\title{
The boundary data immersion method for compressible flows with application to aeroacoustics
}

\author{
Stefan C. Schlanderer ${ }^{\mathrm{a}, 1, *}$, Gabriel D. Weymouth ${ }^{\mathrm{a}, 2}$, Richard D. Sandberg ${ }^{\mathrm{b}, 3}$ \\ ${ }^{a}$ Faculty for Engineering and the Environment \\ University of Southampton, SO17 1BJ Southampton, United Kingdom \\ ${ }^{b}$ Department of Mechanical Engineering, \\ University of Melbourne, Melbourne VIC 3010, Australia
}

\begin{abstract}
This paper introduces a virtual boundary method for compressible viscous fluid flow that is capable of accurately representing moving bodies in flow and aeroacoustic simulations. The method is the compressible extension of the boundary data immersion method (BDIM, Maertens \& Weymouth (2015)). The BDIM equations for the compressible Navier-Stokes equations are derived and the accuracy of the method for the hydrodynamic representation of solid bodies is demonstrated with challenging test cases, including a fully turbulent boundary layer flow and a supersonic instability wave. In addition we show that the compressible BDIM is able to accurately represent noise radiation from moving bodies and flow induced noise generation without any penalty in allowable time step.

Keywords: boundary data immersion, immersed boundary method, aeroacoustics, compressible fluid flow, flow induced noise, noise from moving bodies
\end{abstract}

\section{Introduction}

Accurate computation of fluid flows in the vicinity of moving bodies with high accuracy is a severe challenge. Immersed boundary methods (IMBM)

\footnotetext{
${ }^{*}$ Corresponding author

${ }^{1}$ Present address: stefan.schlanderer@unimelb.edu.au

${ }^{2}$ G.D.Weymouth@soton.ac.uk

${ }^{3}$ richard.sandberg@unimelb.edu.au
} 
have become a popular tool to impose boundary conditions upon boundaries that do not coincide with the computational grid (refer to [2] for a review). Besides the ability to simulate moving bodies this class of boundary methods enables the simulation of complex geometries on relatively simple meshes, thus reducing the effort of grid generation. Alongside with the enormous growth of computational power in recent years IMBMs have enabled the consideration of multi-physics phenomena such as fluid-structure interaction problems. Furthermore flow control and optimization problems incorporating moving control surfaces or morphing bodies can be studied with recent advances of IMBMs.

However, the majority of previously published IMBMs and their extensions considered incompressible fluid flow. For aeroacoustics and compressible fluid flow there are only few methods published, among them two for inviscid flow $[3,4]$. For noise propagation problems with prescribed noise sources Casalino et al.[5] and Arina [6] as well as Cand et al. [7] and Liu \& Wu [8] presented IMBMs in the frequency and time domain, respectively. Hybrid approaches for aeroacoustic research obtain a noise source field from a flow simulation which is then used as input for acoustic analogies to calculate the acoustic farfield. For this class of computational aeroacoustics Seo \& Mittal [9] developed a sharp interface IMBM for the linearized perturbed compressible equations (LPCE). They validated the method with flow induced noise from stationary objects and acoustic scattering problems. Another hybrid approach was employed by Margnat [10] who coupled an incompressible flow solver employing Goldstein's feedback forcing [11] approach with Curle's analogy.

To the authors knowledge all IMBM in the literature for viscous compressible flows were intended and validated to represent stationary bodies $[12$, 1, 13, 14, 15, 16]. Among these studies that consider aeroacoustics Sandberg \& Jones [15] used a ghost cell approach to represent stationary flat plate extensions of airfoils to investigate trailing-edge noise with direct numerical simulations (DNS). Due to the complexity of the boundary reconstruction and the additional treatment for freshly cleared grid points this method would increase computational cost critically for moving boundary problems, rendering it unsuitable for high-fidelity simulations. In the class of continuous forcing methods Liu \& Vasilyev [14] developed a Brinkmann penalization method with a unique density treatment modelling the solid as a high impedance medium for application in aeroacoustic simulations, however without presenting results for flow induced noise. The feedback forcing character of this approach leads to timestep restrictions when using explicit 
time marching which increases computational cost prohibitively.

The "Boundary Data Immersion Method"(BDIM) that has recently been proposed for incompressible simulations by Weymouth \& Yue [17] and extended by Maertens \& Weymouth [18] overcomes many of the aforementioned weaknesses and limitations of existing IMBMs. The concept of this approach is to map the governing equations of the solid body and fluid domain at the interface. This results in a set of meta equations that is valid in both domains and ensures a smooth transition. The smooth transition helps to avoid freshly cleared cell treatment $[19,20,21,22,23]$ that is needed for sharp interface approaches when moving bodies are considered. Furthermore the extension of the BDIM [18] addresses a key problem with smooth IMBMs in representing the discontinuity of the velocity gradient $\frac{\partial u}{\partial y}$ at the wall when considering flows with intermediate to high Reynolds numbers. While the velocity itself is continuous over the interface between fluid and solid due to the no-slip condition the wall normal gradient of the streamwise velocity component is not because of the "kink" in the velocity profile. As the wall velocity gradient increases with Reynolds number this discontinuity becomes more significant [18]. Therefore the error made by momentum forcing approaches increases with an increase in Reynolds number due to the smooth representation of the discontinuity. Furthermore force interpolation and spreading schemes used in momentum forcing approaches assume a smooth field otherwise they become first order accurate or need additional treatment [24]. The higher error and lowered order of accuracy has to be compensated by a finer grid resolution. This typically goes along with a decrease in timestep when using explicit time integration schemes making such a strategy computationally prohibitively expensive. Maertens \& Weymouth [18] increased the accuracy of the mapping between fluid and solid by taking the normal derivative to the surface into account which improved the representation of the jump in the velocity gradient at the wall significantly and lead to a second order convergence rate.

When using a compressible flow solver to directly compute the hydrodynamic and aeroacoustic field simultaneously a method is required that can accurately include the effects of moving bodies and the associated noise generation. Such a method would also enable the consideration of vibro-acoustic problems. To that end the BDIM for the compressible Navier-Stokes equations is derived in this paper in section 2 . The accuracy of the newly devel- 


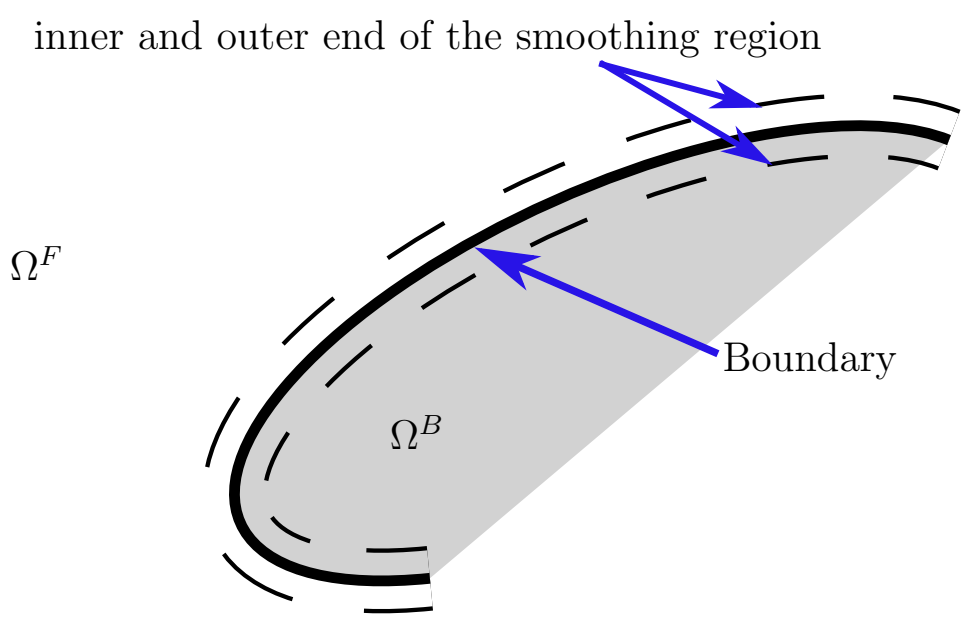

Figure 1: Subdomains under consideration for the derivation of the BDIM equations.

oped method is evaluated on a set of challenging test cases that are highly relevant to aeroacoustic simulations. To the best of the authors' knowledge most of these cases have not been used to validate IMBMs in the past. In section 3 the focus is on the representation of the flow around a cylinder (subsection 3.1) as well as turbulent (subsection 3.2) and supersonic (subsection 3.3) flat plate boundary layers. Section 4 validates the compressible BDIM for the use in aeroacoustic DNS demonstrating its capabilities for problems involving noise generation from moving (subsection 4.1) and stationary objects (subsection 4.2).

\section{Derivation of the compressible flow boundary data immersion equations}

In the following section 2.1 the concept of the BDIM as introduced in [17] and [18] is first revisted. Section 2.2 presents the derivation of the BDIM for the compressible Navier-Stokes equations. Finally the numerical methods of the code into which the compressible BDIM approach was implemented are briefly introduced in section 2.3.

\subsection{The boundary data immersion method revisited}

For the derivation of the BDIM meta equation a domain that incorporates a solid body subdomain $\Omega_{s}$ and a compressible fluid subdomain $\Omega_{f}$ 
are considered, as sketched in Figure 1. The general formulation for a meta equation of an arbitrary field variable $\Phi$ is given by

$$
\Phi_{M}(\vec{x}, t)=\left\{\begin{array}{ll}
\Phi=b & , \text { for } \vec{x} \in \Omega_{b} \\
\Phi=f & , \text { for } \vec{x} \in \Omega_{f}
\end{array} .\right.
$$

Both subdomains can then be smoothly coupled by a convolution of each governing equation with a nascent delta kernel $K_{\epsilon}$ of radius $\epsilon$ which results in

$$
\begin{aligned}
& b_{\epsilon}(\Phi, \vec{x}, t)=\int_{\Omega_{b}} b\left(\Phi, \vec{x}_{b}, t\right) K_{\epsilon}\left(\vec{x}, \vec{x}_{b}\right) \mathrm{d} \vec{x}_{b} \quad \text { and } \\
& f_{\epsilon}(\Phi, \vec{x}, t)=\int_{\Omega_{f}} f\left(\Phi, \vec{x}_{f}, t\right) K_{\epsilon}\left(\vec{x}, \vec{x}_{f}\right) \mathrm{d} \vec{x}_{f}
\end{aligned}
$$

respectively. With this step the domain of each individual governing equation is extended to the whole domain which is the union of both subdomains $\Omega=\Omega_{b} \cup \Omega_{f}$. Hence the governing equation of the field variable $\Phi$ is simply

$$
\Phi_{\epsilon}=b_{\epsilon}+f_{\epsilon} \quad \text { for } \vec{x} \in \Omega \quad \text {. }
$$

The role of the convolution is to "switch off" the governing equations in the complementary subdomain where they are not valid (i.e. fluid equations in solid domain and vice versa). Furthermore it allows a smooth transition between both subdomains over a smoothing region with a half-width of $\epsilon$, where both governing equations contribute to the overall solution. For a simplification of the convolution integrals two requirements are necessary:

1. smoothing only occurs near the boundary/interface

2. smoothing occurs in the normal but not in the tangential direction of the boundary.

Maertens and Weymouth [18] use a Taylor series expansion of the convolution integral and the two aforementioned requirements to derive simplified equations for both extended subdomain equations resulting in

$$
\begin{aligned}
& b_{\epsilon}(\Phi, \vec{x}, t) \approx b(\Phi, \vec{x}, t) \mu_{0}^{\epsilon, B}+\frac{\partial b}{\partial n}(\Phi, \vec{x}, t) \mu_{1}^{\epsilon, B} \quad \text { and } \\
& f_{\epsilon}(\Phi, \vec{x}, t) \approx f(\Phi, \vec{x}, t) \mu_{0}^{\epsilon, F}+\frac{\partial f}{\partial n}(\Phi, \vec{x}, t) \mu_{1}^{\epsilon, F}
\end{aligned}
$$




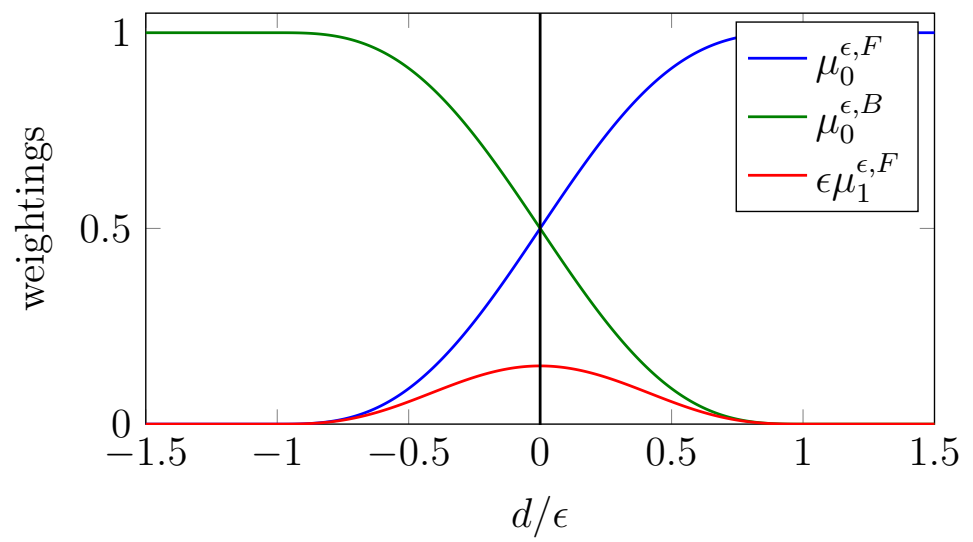

Figure 2: Zeroth and first moment of the kernel for the fluid and solid body domain in the vicinity of the wall surface.

with $\mu_{0}^{\epsilon}$ being the zeroth and $\mu_{1}^{\epsilon}$ first moments of the kernel $\phi_{\epsilon}$ over their respective subdomains. Higher order terms are neglected as they would incorporate terms that are non-local and therefore violate requirement (1). Maertens and Weymouth [18] suggest to use the following kernel

$$
\Phi_{\epsilon}(x, y)= \begin{cases}\frac{1}{2 \epsilon}\left[1+\cos \left(\frac{|x-y| \pi}{\epsilon}\right)\right] & , \text { for }|x-y|<\epsilon \\ 0 & , \text { for }|x-y| \geq \epsilon\end{cases}
$$

which results in the zeroth

$$
\begin{gathered}
\mu_{0}^{\epsilon, F}(d)=\left\{\begin{array}{lll}
\frac{1}{2}\left[1+\frac{d}{\epsilon}+\frac{1}{\pi} \sin \left(\frac{d}{\epsilon} \pi\right)\right] & , \text { for }|d|<\epsilon \\
0 & , \text { for } d \leq-\epsilon & \text { and first } \\
1 & , \text { for } d \geq \epsilon
\end{array}\right. \\
\mu_{1}^{\epsilon, F}(d)= \begin{cases}\epsilon\left[\frac{1}{4}\left(1-\frac{d^{2}}{\epsilon}\right)-\frac{1}{2 \pi}\left(\frac{d}{\epsilon} \sin \left(\frac{d}{\epsilon} \pi\right)+\frac{1}{\pi}\left(1+\cos \left(\frac{d}{\epsilon} \pi\right)\right)\right)\right] & , \text { for }|d|<\epsilon \\
0 & , \text { for }|d| \geq \epsilon\end{cases}
\end{gathered}
$$

moment of the kernel 5, respectively, and are visualized in figure 2. In these expressions for the zeroth and first kernel moment, $d$ is the signed distance from the surface where values with $d<0$ refer to the inside and $d>0$ to the outside of the solid body. For the solid body domain the symmetry of the 
kernel functions can be used resulting in $\mu_{0}^{\epsilon, B}(d)=\mu_{0}^{\epsilon, F}(-d)=1-\mu_{0}^{\epsilon, F}(d)$ and $\mu_{1}^{\epsilon, B}(d)=\mu_{1}^{\epsilon, F}(-d)=-\mu_{1}^{\epsilon, F}(d)$, respectively [18]. Using equation 3 with 4 and the kernel moments from equation 6 the resulting meta equation can be assembled and results in

$$
\begin{aligned}
\Phi_{\epsilon} & =f(\Phi, \vec{x}, t) \mu_{0}^{\epsilon}+b(\Phi, \vec{x}, t)\left(1-\mu_{0}^{\epsilon}\right) \\
& +\mu_{1}^{\epsilon} \frac{\partial}{\partial n}(f(\Phi, \vec{x}, t)-b(\Phi, \vec{x}, t))
\end{aligned}
$$

This meta equation will be used in the following section to derive the BDIM equations for compressible flows. In Maertens \& Weymouth [18] the derivative correction term determined the order of accuracy of the method when $\epsilon$ and the grid was refined. In this paper results will be denoted "first order" when this term is not included in equation 7 , hence $\mu_{1}^{\epsilon}=0$. The framework will be called second order when the derivative term is included.

In the vicinity of sharp edges of a body surface, e.g. a rectangular trailingedge, the kernel moments need to be interpolated between two line segments in order to properly account for the contribution of both surfaces. To that end we use the interpolation approach detailed in AppendixA.

\subsection{The Boundary Data Immersion Method for the Compressible Navier- Stokes Equations}

In order to use the BDIM meta equation in a compressible flow solver, the compressible Navier-Stokes equations governing the fluid need to be mapped to the governing equation of the solid body. The non-dimensional conservative fluid equations in tensor notation can be expressed with

$$
\begin{aligned}
\frac{\partial \rho}{\partial t}+\frac{\partial}{\partial x_{k}}\left(\rho u_{k}\right) & =0 \\
\frac{\partial}{\partial t}\left(\rho u_{i}\right)+\frac{\partial}{\partial x_{k}}\left(\rho u_{i} u_{k}\right) & =-\frac{\partial p}{\partial x_{k}} \delta_{i k}+\frac{\partial}{\partial x_{k}} \tau_{i k}, \\
\frac{\partial}{\partial t}(\rho E)+\frac{\partial}{\partial x_{k}}\left(\rho u_{k} E\right) & =\frac{\partial}{\partial x_{k}}\left[u_{i} \tau_{i k}-u_{k} p-q_{k}\right] .
\end{aligned}
$$

In this set of equations the conservation of mass is expressed by $8 \mathrm{a}$, conservation of momentum by $8 \mathrm{~b}$ and finally conservation of total energy $(\rho E)$ by $8 \mathrm{c}$. The fluid velocity is denoted by $u_{i}$, density by $\rho$, static pressure by $p$ and the temperature by $T$. The total energy $E$ is defined as $E=$ $T /\left[\gamma(\gamma-1) M^{2}\right]+1 / 2 u_{i} u_{i}$ and the stress tensor and heat-flux vector are 
computed as

$$
\tau_{i k}=\frac{\mu}{R e}\left(\frac{\partial u_{i}}{\partial x_{k}}+\frac{\partial u_{k}}{\partial x_{i}}-\frac{2}{3} \frac{\partial u_{k}}{\partial x_{k}} \delta_{i k}\right) \quad \text { and } \quad q_{k}=\frac{-\mu}{(\gamma-1) M^{2} \operatorname{Pr} R e} \frac{\partial T}{\partial x_{k}}
$$

respectively, where $M$ is the Mach number, $R e$ the Reynolds number and $\operatorname{Pr}$ the Prandtl number which is assumed to be constant at $\operatorname{Pr}=0.72$. The Mach and Reynolds numbers are a result of the non-dimensionalization. Finally the system of equations is closed by obtaining the pressure $p$ with the non-dimensional equation of state $p=(\rho T) /\left(\gamma M^{2}\right)$ where $\gamma=1.4$ is the isentropic exponent of air.

For the introduction of the governing equation of the solid body the dependence of all quantities on the location is neglected in the notation throughout the following paragraphs. Nevertheless all quantities can vary in space. The conservation of mass for a solid results in the same expression as for a compressible fluid, namely

$$
\frac{\partial \rho}{\partial t}+\frac{\partial}{\partial x_{k}}\left(\rho u_{k}\right)=0
$$

Furthermore the solid body has a prescribed velocity $V(\vec{x}, t)$ which can be provided a priori with an analytical equation or a time dependent solution from a structural solver or a flow control framework. In addition the temperature $T_{s}$ within the solid body domain is prescribed as a constant or by another input function. Hence the governing equation for the velocity $b_{V}$ and temperature $b_{T}$ of the solid body are

$$
\begin{aligned}
b_{V_{i}} & =u_{i, b}(t)=V_{s, i}(t) \quad \text { and } \\
b_{T} & =T_{b}(t)=T_{s}(t)
\end{aligned}
$$

Since the conservation of mass exploits the same physical principle and has the same expression in both subdomains no mapping of the governing equations is needed.

Before the velocity equation 11a of the solid can be mapped to the momentum equation $8 \mathrm{~b}$ of the fluid, both equations need to be reformulated in order to describe the same physical quantities. The left-hand side of equation $8 \mathrm{~b}$ 
can be expanded as

$$
\begin{aligned}
\frac{\partial}{\partial t}\left(\rho u_{i}\right)+\frac{\partial}{\partial x_{k}}\left(\rho u_{i} u_{k}\right) & =u_{i} \frac{\partial \rho}{\partial t}+\rho \frac{\partial u_{i}}{\partial t}+\rho u_{k} \frac{\partial u_{i}}{\partial x_{k}}+u_{i} \frac{\partial \rho u_{k}}{\partial x_{k}} \\
& =\rho\left[\frac{\partial u_{i}}{\partial t}+u_{k} \frac{\partial u_{i}}{\partial x_{k}}\right]
\end{aligned}
$$

The sum of the first and the last term of right-hand-side of the expanded equation are the continuity equation multiplied by $u_{i}$ and therefore are zero. Hence, after some rearrangement with the right-hand side from equation $8 \mathrm{~b}$ the change of the fluid velocity in time is

$$
\frac{\partial u_{i}}{\partial t}=-u_{k} \frac{\partial u_{i}}{\partial x_{k}}+\frac{1}{\rho}\left[-\frac{\partial p}{\partial x_{k}} \delta_{i j}+\frac{\partial}{\partial x_{k}} \tau_{i j}\right]=R H S_{u, i}
$$

$R H S_{u, i}$ will be used as an abbreviation for the right-hand-side of the velocity equation in the following derivation. The expression for the velocity field can then be obtained by integrating this equation over a time step $\Delta t$ resulting in

$$
f_{v}\left(u_{i}, t_{0}+\Delta t\right)=u_{i, f}\left(t_{0}+\Delta t\right)=u_{i, f}\left(t_{0}\right)+\int_{t_{0}}^{t_{0}+\Delta t} R H S_{u, i} d t
$$

With an Euler forward integration or a generalized Runge-Kutta substep in time this equation can be discretized with

$$
f_{v}\left(u_{i}, t_{0}+\Delta t\right)=u_{i, f}\left(t_{0}+\Delta t\right)=u_{i, f}\left(t_{0}\right)+R H S_{u, i} \Delta t \quad .
$$

Since the governing equations for the velocity in both subdomains are now defined with equation $11 \mathrm{a}$ and 15 they can be replaced in 7 which leads to

$$
\begin{aligned}
u_{i, \epsilon}\left(t_{0}+\Delta t\right) & =\left[u_{i, f}\left(t_{0}\right)+R H S_{u, i} \Delta t\right] \mu_{0}^{\epsilon}+V_{i}(t)\left(1-\mu_{0}^{\epsilon}\right) \\
& +\mu_{1}^{\epsilon} \frac{\partial}{\partial n}\left[u_{i, f}\left(t_{0}\right)+R H S_{u, i}-V_{i}(t)\right]
\end{aligned}
$$

In contrast to incompressible fluid solvers where the pressure equation is commonly solved separately from the rest of the right hand side of equation 13 , all of these terms are commonly treated equally in compressible frameworks. Therefore the mapped velocity field of the fluid can be summarized 
with its integral formulation which results in

$$
u_{i, \epsilon}(t)=u_{i, f}(t) \mu_{0}^{\epsilon}+V_{i}(t)\left(1-\mu_{0}^{\epsilon}\right)+\mu_{1}^{\epsilon} \frac{\partial}{\partial n}\left[u_{i, f}(t)-V_{i}(t)\right] \quad .
$$

Following the same approach as for the velocity equation the left hand side of the total energy equation $8 c$ can be expanded as

$$
\frac{\partial}{\partial t}(\rho E)+\frac{\partial}{\partial x_{k}}\left(\rho u_{k} E\right)=E \frac{\partial \rho}{\partial t}+\rho \frac{\partial E}{\partial t}+\rho u_{k} \frac{\partial E}{\partial x_{k}}+E \frac{\partial \rho u_{k}}{\partial x_{k}} \quad .
$$

The sum of the first and the last terms of the right-hand-side of the expanded equation are the continuity equation multiplied by the total energy $E$ and hence add up to zero. By substituting the definition of the total energy $E=$ $T /\left[\gamma(\gamma-1) M^{2}\right]+1 / 2 u_{i} u_{i}$ and using the abbreviation $T /\left[\gamma(\gamma-1) M^{2}\right]=\chi$ this yields

$$
\begin{aligned}
\rho \frac{\partial E}{\partial t}+\frac{\partial}{\partial x_{k}}\left(\rho u_{k} E\right) & =\rho\left[\chi \frac{\partial T}{\partial t}+\frac{1}{2} \frac{\partial u_{i} u_{i}}{\partial t}\right]+\rho u_{k}\left[\rho \chi \frac{\partial T}{\partial x_{k}}+\frac{1}{2} \frac{\partial u_{i} u_{i}}{\partial x_{k}}\right] \\
& =\rho\left[\chi \frac{\partial T}{\partial t}+u_{i} \frac{\partial u_{i}}{\partial t}\right]+\rho u_{k}\left[\chi \frac{\partial T}{\partial x_{k}}+u_{i} \frac{\partial u_{i}}{\partial x_{k}}\right] \\
& =\rho\left[\chi \frac{\partial T}{\partial t}+u_{k} \chi \frac{\partial T}{\partial x_{k}}\right]+\rho u_{i}\left[\frac{\partial u_{i}}{\partial t}+u_{k} \frac{\partial u_{i}}{\partial x_{k}}\right]
\end{aligned}
$$

The two terms in the final brackets of the last line are the left hand side of the expanded momentum equation 12 multiplied by $u_{i}$. They represent the change of kinetic energy of the fluid after applying the chain rule. Using that fact together with the right hand side of equation $8 \mathrm{c}$, the change of temperature in time can be expressed by

$$
\begin{aligned}
\frac{\partial T}{\partial t} & =-u_{k} \frac{\partial T}{\partial x_{k}}+\frac{1}{\rho \chi} \frac{\partial}{\partial x_{k}}\left[u_{i} \tau_{i k}-u_{k} p-q_{k}+u_{i} p \delta_{i k}-u_{i} \tau_{i k}\right] \\
& =-u_{k} \frac{\partial T}{\partial x_{k}}+\frac{1}{\rho \chi} \frac{\partial}{\partial x_{k}}\left[-u_{k} p-q_{k}+u_{i} p \delta_{i k}\right]=R H S_{T}
\end{aligned}
$$

Analogous to the time integration of the velocity field the temperature at time $t=t_{o}+\Delta t$ is

$$
f_{T}\left(T, t_{0}+\Delta t\right)=T_{f}\left(t_{0}+\Delta t\right)=T_{f}\left(t_{0}\right)+R H S_{T} \Delta t
$$


and can be substituted into the general meta equation together with the governing equation for the solid's temperature $11 \mathrm{~b}$

$$
\begin{aligned}
T_{\epsilon}\left(t_{0}+\Delta t\right) & =\left[T_{f}\left(t_{0}\right)+R H S_{T} \Delta t\right] \mu_{0}^{\epsilon}+T_{s}(t)\left(1-\mu_{0}^{\epsilon}\right) \\
& +\mu_{1}^{\epsilon} \frac{\partial}{\partial n}\left[T_{f}\left(t_{0}\right)+R H S_{T} \Delta t-T_{s}(t)\right] .
\end{aligned}
$$

With the same reasoning that was used for the velocity field the BDIM equation for temperature can be simplified with its integral formulation

$$
T_{\epsilon}\left(t_{0}+\Delta t\right)=T_{f}(t) \mu_{0}^{\epsilon}+T_{s}(t)\left(1-\mu_{0}^{\epsilon}\right)+\mu_{1}^{\epsilon} \frac{\partial}{\partial n}\left[T_{f}(t)-T_{s}(t)\right] \quad .
$$

As the resulting BDIM equations can all be expressed with the integral formulation the boundary data immersion method can be applied after the time integration and no adaptation of the original time-stepping algorithm for the right hand side is needed. Furthermore this is consistent with the application of body-fitted boundary conditions which are commonly performed at the same stage of the simulation.

A comparison between the derived meta equations 17 and 23 and discrete momentum forcing reveals that the first order BDIM is very similar to discrete momentum forcing. When the $\mu_{0}^{\epsilon}$ and $1-\mu_{0}^{\epsilon}$ terms are regarded as the interpolation and force spreading operators, then the only difference between both approaches is the physical reasoning for using them and how these operators work. The analytical and general reasoning, however, is a strength of the BDIM approach, offering robust and smooth coupling where other approaches such as discrete momentum forcing need additional treatment for moving bodies. In addition the second order extension of the approach offers higher order interpolation between both subdomains and reduces bias of the velocity gradient discontinuity.

In the incompressible meta equations derived in [17] and [18] there is an additional weighting for the pressure term as a boundary condition for the pressure equation. This is a result of the special algorithmic treatment of the pressure by the Poisson equation in incompressible flow simulations, which is in principle not required in the present compressible framework. However, it was found that for bluff bodies where the body surface alignment with the underlying grid is arbitrary, instabilities on single grid points could grow just inside the smoothing region. These instabilities would eventually lead to diverging simulations. Analysis of the contributing terms of the continuity 
equation

$$
\frac{\partial \rho}{\partial t}=-\frac{\partial}{\partial x_{k}}\left(\rho u_{k}\right)=-\left(\rho \frac{\partial u_{k}}{\partial x_{k}}+u_{k} \frac{\partial \rho}{\partial x_{k}}\right)
$$

at these grid points showed that the instabilities were caused by the bias in the derivative terms of the velocity, i.e. the first term which contains the incompressible contribution. In particular the derivative in the wall tangential direction lead on average to a non-zero right-hand side of the continuity equation. In order to ensure robust simulations without prescribing something unphysical it is suggested to map the right hand side of equation 24 to

$$
\frac{\partial \rho}{\partial t}=-\left(\rho \frac{\partial u_{n}}{\partial n}\right)
$$

Here the subscript and direction $n$ mean the wall normal direction. This is exactly the simplified right hand side of the continuity equation at the wall for a stationary body. In this case the second term of equation 24 is zero and the only non-zero component of the first term is the one in the wall normal direction. Strictly speaking equation 25 is not the governing equation of the solid interior but an interface condition. Replacing equation 24 and 25 in the general meta equation 7 results in

$$
\frac{\partial \rho_{\epsilon}}{\partial t}=-\frac{\partial\left(\rho u_{k}\right)}{\partial x_{k}} \mu_{0}^{\epsilon}-\left(\rho \frac{\partial u_{n}}{\partial n}\right)\left(1-\mu_{0}^{\epsilon}\right)-\mu_{1}^{\epsilon} \frac{\partial}{\partial n}\left(\frac{\partial\left(\rho u_{k}\right)}{\partial x_{k}}-\left(\rho \frac{\partial u_{n}}{\partial n}\right)\right)
$$

as meta equation for the right-hand of the continuity equation.

\subsection{Numerical methods to solve the compressible Navier-Stokes equations}

The code that was used to validate the compressible BDIM solves the Navier-Stokes equations employing a $4^{\text {th }}$-order accurate standard-difference scheme with Carpenter boundary stencils [25] to calculate derivatives. The scheme can be applied to general geometries by pre-multiplication of metric terms. Time marching is achieved employing an ultra low-storage five-step $4^{\text {th }}$-order accurate Runge-Kutta scheme [26]. The stability of the code is enhanced by a skew-symmetric splitting of the nonlinear terms [27]. Furthermore a sixth-order accurate high-wavenumber cut-off filter [28] with a weighting of $0.1-0.2$ is employed after every full Runge-Kutta cycle. Unphysical numerical reflections at the domain boundaries are avoided by using characteristic boundary conditions. At the inflow they are applied in an integral formulation as described by Jones [29]. For the outflow a zonal characteristic 
boundary condition [30] is used to avoid spurious pressure-oscillations from the outflow boundary which is subject to the passage vortical structures.

\section{Validation for hydrodynamic accuracy}

\subsection{Flow around cylinder}

The canonical flow around a static cylinder at Reynolds number $R e_{D_{c y l}}=$ 100 is considered first as a validation case which offers a large number of reference data in the literature. The Mach number was set to $M=0.1$, thus compressibility effects are expected to be negligible. The flow is simulated in a domain where the cylinder is placed at the origin and the domain boundaries are $-27 D_{c y l}<x<30 D_{c y l}$ and $-30 D_{c y l}<y<30 D_{c y l}$. The cylinder is immersed in a fluid flow with a streamwise velocity of unity which is prescribed at the inflow. The upper and lower boundaries are modelled as non-reflective characteristic freestream boundary conditions. At the outflow boundary the zonal characteristic boundary condition proposed in [30] is employed. The grid is spaced uniformly in the $x$ and $y$ directions within and a region of $-2 R_{c y l}<x<2 R_{c y l}$ and $-2 R_{c y l}<y<2 R_{c y l}$. The uniform grid spacing was $\Delta x=D_{\text {cyl }} / 120$, thus resulting in 180 points within the equidistant discretization. The grid was then stretched towards the boundaries over 95 grid points with polynomial functions. The smoothing region half-width is set to $\epsilon=2 \Delta x=2 \Delta y$. The mapping of the continuity equation as introduced in equation 26 is employed in that case.

\subsubsection{Assessment of Accuracy}

The forces acting on the cylinder are calculated from the pressure and skin-friction values on the surface of the cylinder. To that end the cylinder surface was discretized by 360 points equally distributed over the circumference. Since the values within the smoothing region are not physical the surface quantities were evaluated with a distance of $\epsilon$. The accuracy of the pressure force is expected to not be affected by that. However, the skinfriction will be underestimated with this method. This is not a specific problem related to the BDIM but occurs in other IMBM approaches as well [39]. The relevant flow quantities, i.e pressure and wall normal velocity gradient are interpolated from the four surrounding fluid grid points onto the surface grid using bilinear interpolation as described in [40].

The final values for the lift and drag are obtained by a panel integration over all surface points. The results for the Strouhal number, the lift fluctuations 


\begin{tabular}{cccccccc}
\hline \hline Source & $S t$ & $C_{D}$ & $C_{L}$ & $C_{D p}$ & $C_{D f}$ & $C_{L p}$ & $C_{L f}$ \\
\hline Exp. & 0.164 & 1.25 & - & - & - & - & - \\
\hline$[31]$ & 0.165 & $1.33 \pm 0.009$ & \pm 0.3321 & $0.99 \pm 0.0082$ & \pm 0.0010 & \pm 0.295 & \pm 0.042 \\
{$[32]$} & & 1.33 & & 1.00 & 0.32 & - & - \\
{$[33]$} & 0.172 & 1.42 & \pm 0.3536 & - & - & - & - \\
\hline & & & & & & & - \\
{$[34]$} & 0.164 & $1.34 \pm 0.011$ & \pm 0.315 & - & - & - & - \\
{$[35]$} & 0.167 & $1.35 \pm 0.012$ & \pm 0.315 & - & - & - & - \\
{$[36]$} & 0.165 & $1.34 \pm 0.007$ & \pm 0.276 & - & -1.035 \\
{$[18] 1$ st order } & 0.167 & $1.31 \pm 0.009$ & \pm 0.321 & $1.01 \pm 0.0085$ & $0.30 \pm 0.0008$ & \pm 0.292 & \pm 0.035 \\
{$[18] 2$ nd order } & 0.167 & $1.31 \pm 0.009$ & \pm 0.313 & $1.00 \pm 0.0081$ & $0.30 \pm 0.0007$ & \pm 0.285 & \pm 0.034 \\
1st order & 0.165 & $1.35 \pm 0.011$ & \pm 0.348 & $1.03 \pm 0.0104$ & $0.32 \pm 0.0010$ & \pm 0.314 & \pm 0.040 \\
2nd order & 0.165 & $1.32 \pm 0.011$ & \pm 0.333 & $1.02 \pm 0.0010$ & $0.30 \pm 0.0008$ & \pm 0.304 & \pm 0.036 \\
& & & & & & & \\
\hline \hline
\end{tabular}

Table 1: Summary of the results from the cylinder case employing the first and second order BDIM to represent the cylinder shape for the baseline resolution of $\Delta x=D_{c y l} / 120$. The results are compared to experimental data ( $S t$ from [37] with estimated uncertainty of $0.8 \%, C_{D}$ from [38] with estimated measurement error of $6 \%$ ), body-fitted simulations [31, $32,33]$ and other IMBMs $[34,35,36,18]$.

as well as the drag are presented in table 1 . They are presented alongside data available in the literature from experiments [37, 38], body-fitted simulations $[31,32,33]$ and other IMBMs [34, 35, 36, 18]. Given the scatter among the reported results the data obtained from simulations with the BDIM agree reasonably well with the values from literature.

Finally the pressure and wall vorticity distribution on the cylinder surface are presented as a function of the azimuthal position and compared to reference [31] in figure 3. The distribution of the pressure coefficient $C_{p}$ in figure 3a shows excellent agreement with the reference for the first and the second order BDIM frame work. The skin friction was calculated as

$$
C_{f}=\frac{2 \nu}{U_{\infty}^{2}} \frac{\overrightarrow{u_{\epsilon}}(\vec{x}+\epsilon \hat{n}) \cdot \hat{t}}{\epsilon},
$$

with the kinematic viscosity $\nu$ and the freestream velocity $U_{\infty}^{2}$. The wall normal and tangent unit vector are denoted $\hat{n}$ and $\hat{t}$, respectively. Figure $3 \mathrm{~b}$ compares the skin friction distribution of the two BDIM frameworks to reference [31]. It is found that the first order BDIM underestimates the skin friction significantly. However, when the wall normal derivative correction is 


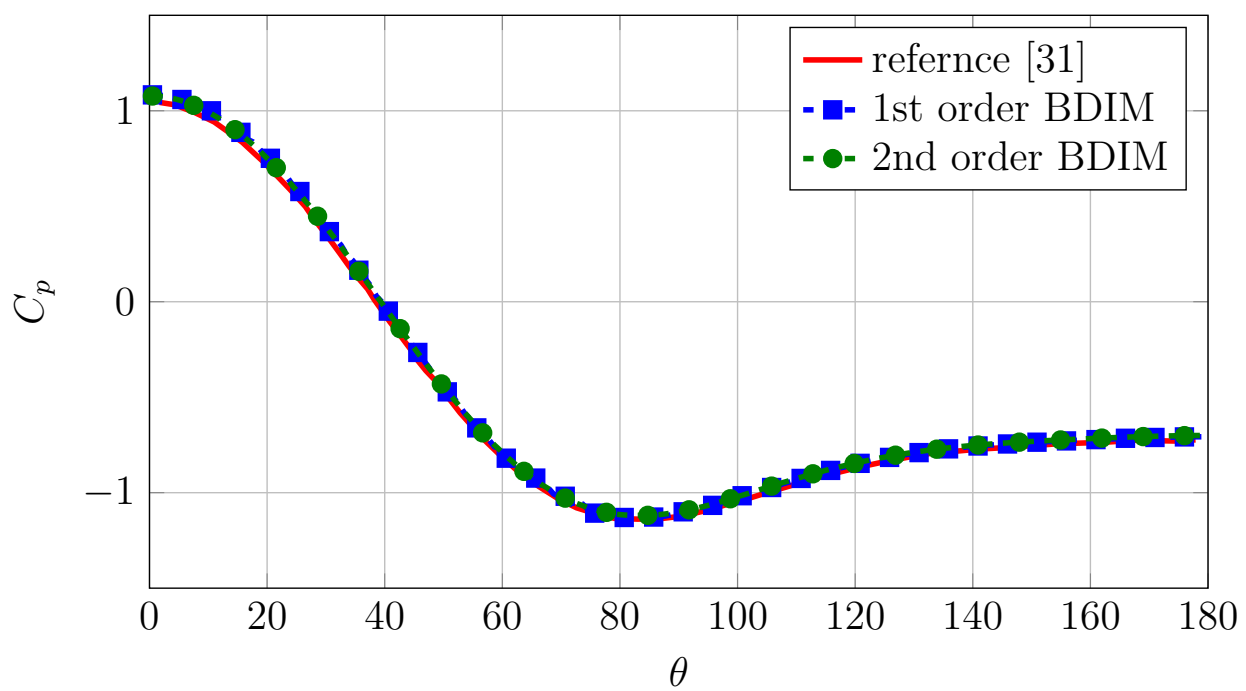

(a) Pressure distribution on the cylinder for the first and second order BDIM in comparison with the reference solution [31].

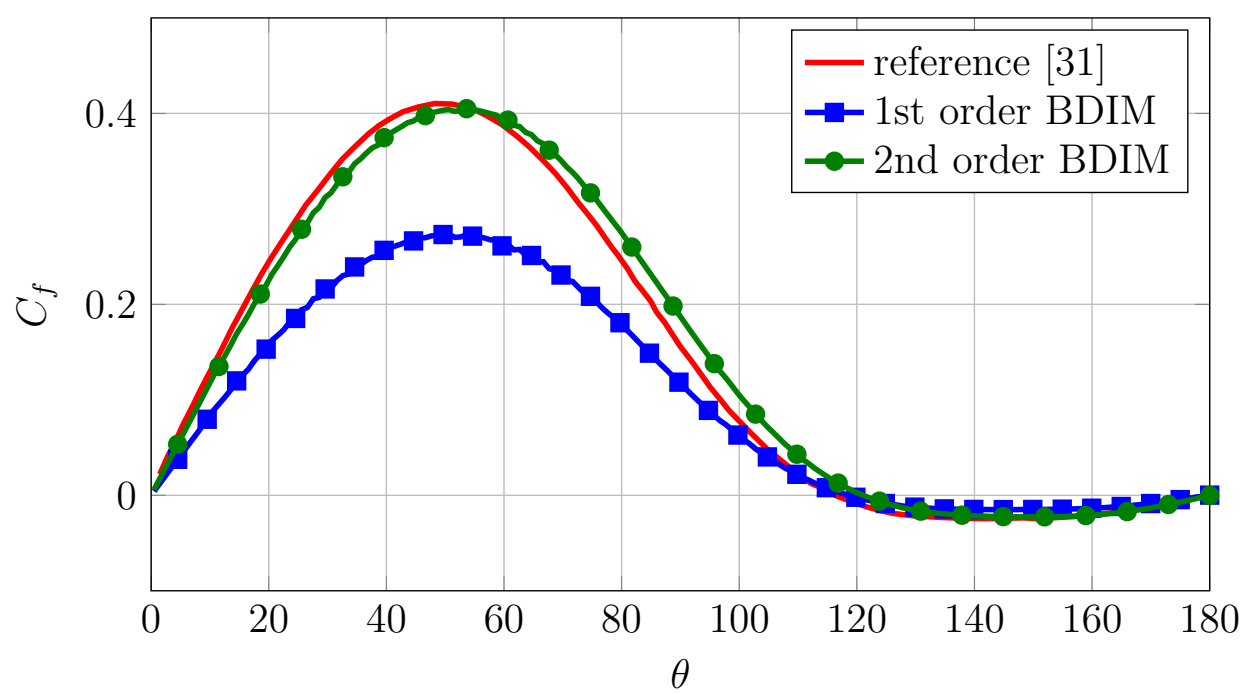

(b) Spanwise wall vorticity distribution on the cylinder comparing the first and second order BDIM with reference data [31].

Figure 3: Comparison of the pressure and skin friction distribution on the surface of the cylinder. 
employed the maximum distribution is predicted very accurately.

\subsubsection{Formal order of convergence}

In order to evaluate the formal order of convergence for the numerical setup the simulation of the flow around the cylinder was repeated on six grids that were coarser than the one of the baseline simulation from the previous section. The resolution levels are defined in multiples $n$ of the baseline resolution with $n D_{c y l} / 120=[3,4,6,8,10]$. The computational cost for the study was reduced by setting the Mach number to $M=0.3$ which allowed an increase of the time step of the simulation. Since there is no analytical solution for this flow problem the data from the highly resolved case presented in the previous section with a grid spacing of $\Delta x=\Delta y=$ $D_{\text {cyl }} / 120$ was used as a reference.

Commonly the $L_{2}$ norm is considered as a measure for the global error made by the boundary scheme. The $L_{\infty}$ on the other hand highlights the local error close to the boundary. Figure 4 presents the results for the convergence study in the $L_{2}$ (4a and $4 \mathrm{~b}$ ) as well as in the $L_{\infty}$ (4c and $4 \mathrm{~d}$ ) norm for the velocity and pressure fields over the different grid resolutions. In general it can be found that the results with the first order framework always yield an error that is higher than that obtained with the second order correction. For the $L_{2}$ and $L_{\infty}$ norms the difference in error of the velocity field from the first order approach is a factor of $2.6-8.6$ higher than that of the second order BDIM. For the pressure field the differences are a bit lower but still significant with a factor of $2.0-3.0$. Each plot of figure 4 also shows first and second order slopes. Comparing these to the convergence data one can find that the first order approach yields a convergence rate that is slightly higher than first order for the velocity field (4a and 4c) in both the $L_{2}$ and $L_{\infty}$ norm. In contrast to that the second order framework shows second order convergence approximately in the $L_{2}$ and $L_{\infty}$ norm. For the pressure field the convergence rates of both approaches are approximately the same with second order for the higher resolution levels and slightly lower for the coarsest two.

\subsection{Turbulent Boundary layer}

In many engineering applications featuring fluid flow with moving or stationary solid objects the flow is turbulent. For IMBMs the main challenge in representing such flows is the high velocity gradient at the wall which increases with Reynolds number. As a result of that the discontinuity in the 


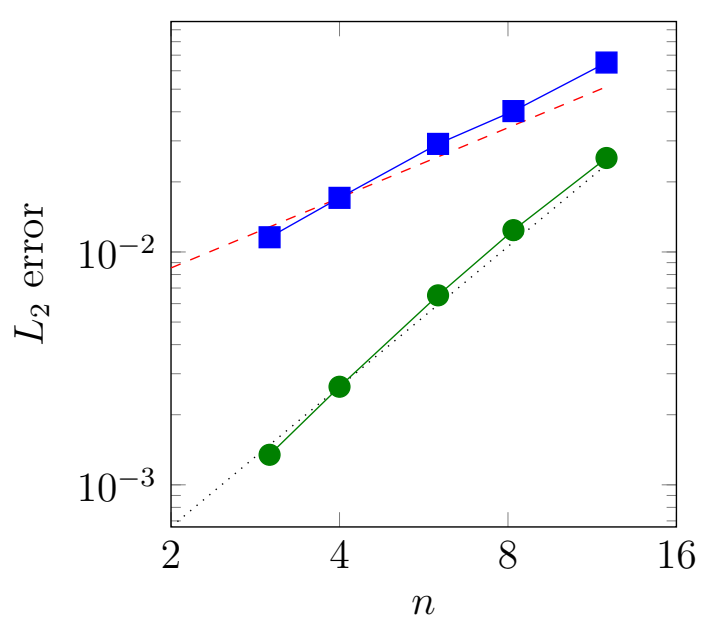

(a) $L_{2}(u)$

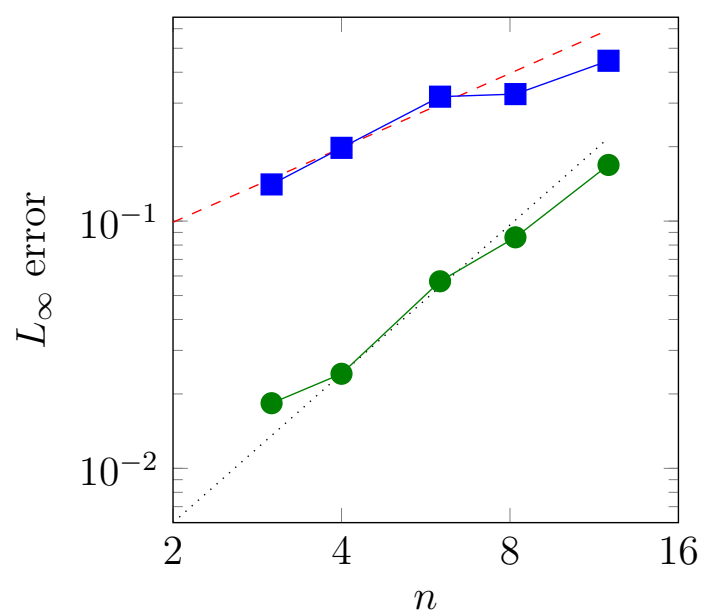

(c) $L_{\infty}(u)$

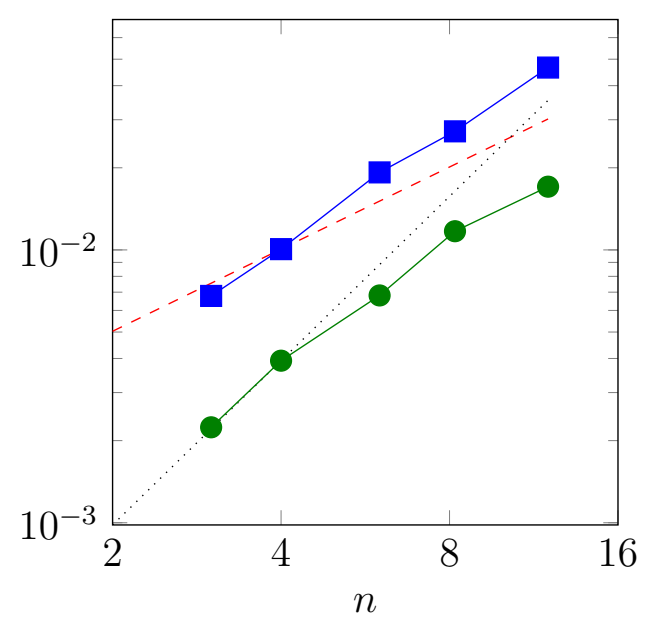

(b) $L_{2}(p)$

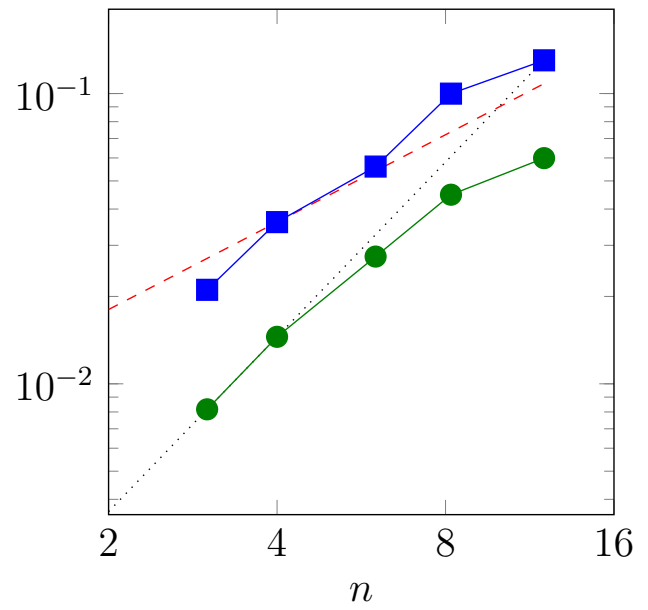

(d) $L_{\infty}(p)$

\section{- - first order …... second order - - 1 st order BDIM $\bullet-2$ nd order BDIM}

Figure 4: $L_{2}$ and $L_{\infty}$ convergence of the velocity and pressure field for the flow around a stationary cylinder. A first and second order slope are shown as reference. 
velocity gradient profile at the wall will grow as well. In particular aeroacoustic noise generation from the interaction of fluid flow with solid objects such as turbulent interaction or trailing-edge noise is very sensitive to an accurate representation of the physics in the near wall region. The fully turbulent flow over a flat plate is used to assess the accuracy of the BDIM in representing the near wall physics in the presence of the relatively high velocity gradient discontinuity and to assess the BDIM's performance for problems featuring a large range of temporal and spatial scales.

\subsubsection{Numerical Setup}

The domain for the turbulent boundary layer incorporated the streamwise range of $1073<R e_{\theta}<1595$ where $R e_{\theta}$ is the Reynolds number based on momentum thickness. The wall normal grid spacing was constant along the streamwise direction. When scaled with the local viscous units the maximum spacing was $\Delta y^{+}=0.97$. In the wall normal direction the grid was stretched towards the freestream boundary over 90 grid points to $\Delta y^{+}=106$ at a distance of $20.6 \delta^{*}$, where $\delta^{*}$ is the displacement thickness at the outflow. The grid spacing in the streamwise direction was uniform with maximum value of $\Delta x^{+}=18.0$ when scaled with the local viscous units. The spanwise domain width was $l_{z}=5 \delta^{*}$. A spectral method with 32 modes was used to discretize the domain width which yields a spanwise grid spacing of $\Delta z^{+}=8.13$. At the inflow synthetic turbulent fluctuations were superimposed to a mean turbulent velocity profile. To that end random fluctuations were filtered using the digital filter method proposed by Klein et al. [41] and extended to compressible flows by Touber \& Sandham [42]. This method has been shown to result in good predictions of second order moments such as velocity fluctuations and integral turbulent length scales downstream of a development region with roughly $20 \delta$ length. The freestream and outflow boundary condition were both prescribed using characteristic boundary conditions. At the outflow the zonal approach was employed [30].

When the BDIM was employed to impose the wall boundary condition 20 grid points were mirrored below the actual surface of the wall and initialized with zero velocity, uniform density and the wall temperature.

\subsubsection{Results}

One of the main challenges for immersed boundary methods that do not modify the discretization at the boundary by reconstructing the boundary is 

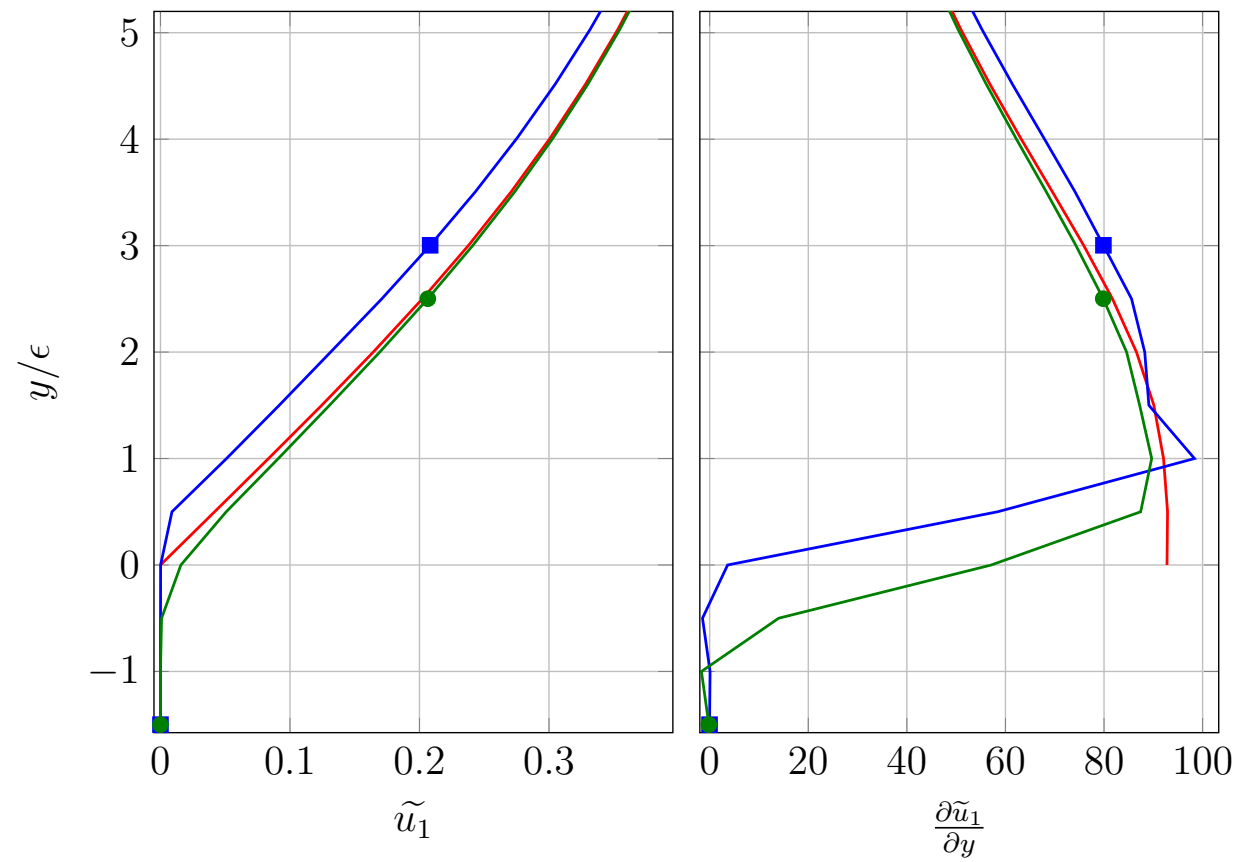

body-fitted $\rightarrow-1$ st order BDIM $\bullet-2$ nd order BDIM

Figure 5: Streamwise velocity profile and its gradient in wall normal direction over the wall normal distances normalized by the smoothing region halfwidth $\epsilon$ in direct vicinity of the wall for the turbulent boundary layer flow at $R e_{\theta} \approx 1410$.

posed through the discontinuity of the wall velocity gradient $\frac{\partial u_{1}}{\partial y}$. Figure 5 presents the streamwise velocity profile and its wall normal gradient in direct vicinity of the wall for the body-fitted and the two BDIM simulations. The kink and discontinuity in the velocity profile and its derivative, respectively, are immediately apparent in the body-fitted simulation. The results obtained with the first order BDIM show that the velocity profile is offset towards the free stream at all wall normal locations. Furthermore the smoothing region of halfwidth $\epsilon=2 \Delta y$ is hardly used to transition from the governing equations of the solid body to the fluid. In contrast, the second order BDIM features a much smoother transition between the two sets of governing equations and also takes advantage of a larger fraction of the smoothing region. In that case the deviation in the velocity profile from the body-fitted reference is very small at the boundary of the smoothing region and can be neglected at greater wall distances. 


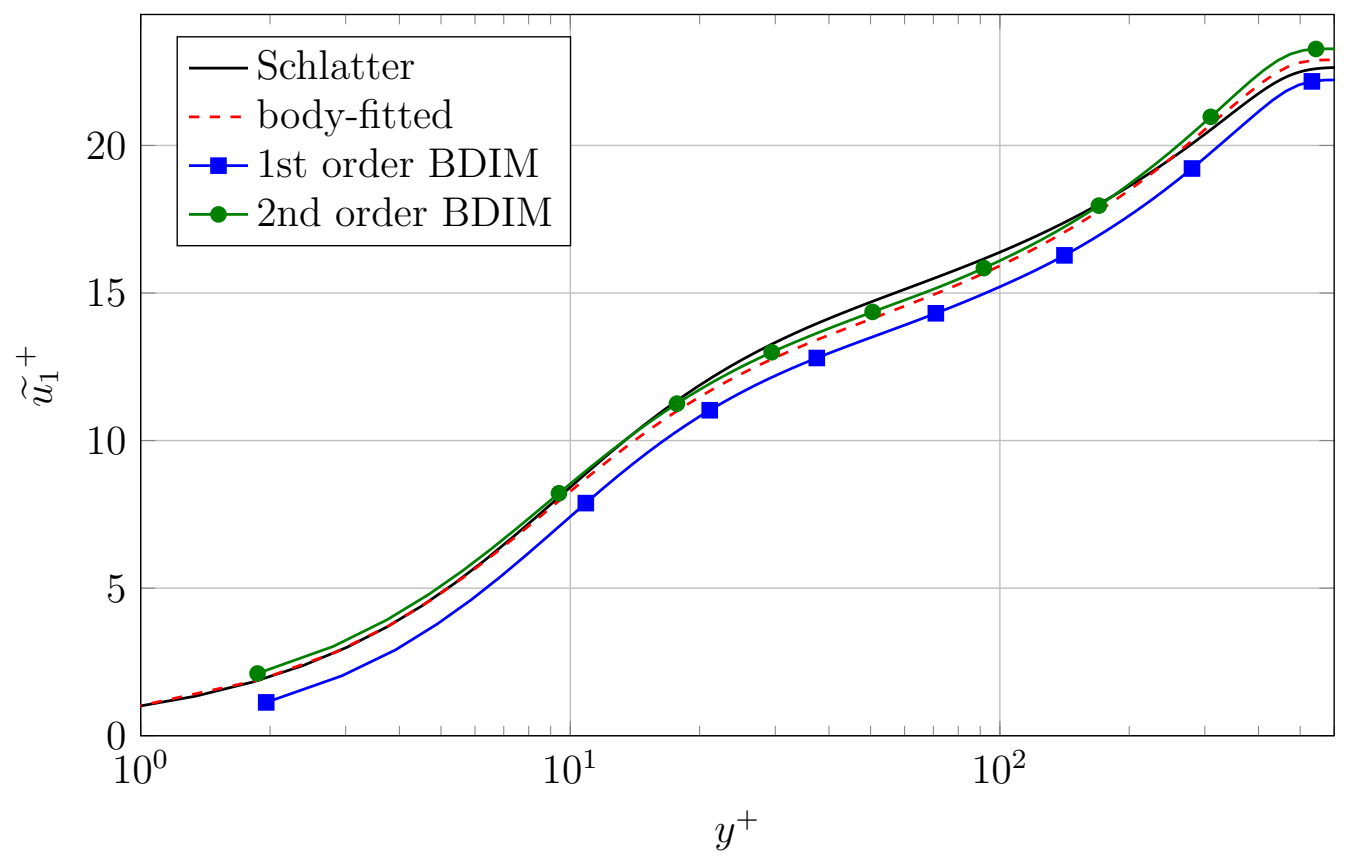

Figure 6: Turbulent boundary layer velocity profile scaled with viscous units comparing the BDIM to the body-fitted boundary conditions and the reference data from Schlatter and Örlü [43]. The normalization of the velocity profiles from the BDIM simulations was calculated with $\frac{\partial \widetilde{u_{1}}}{\partial y}$ at $y=\epsilon$.

Considering the wall normal derivative of the velocity profile it can be found that the first order BDIM introduces overshoots towards the solid body and the fluid. The maximum overshoot is at the fluid boundary of the smoothing region and significant deviations from the reference as well as an offset towards the freestream can be observed. When the second order correction is employed there is only a single overshoot towards the solid and the discontinuity is bridged in a considerably smoother fashion. The difference to the reference at the fluid boundary is fairly small and vanishes towards the freestream.

Maertens and Weymouth [18] found qualitatively the same behaviour for a laminar channel and showed that the use of the wall normal derivative lead to second order convergence in the $L_{2}$ and $L_{\infty}$ norm.

Turbulent quantities are commonly normalized by viscous scales derived from the friction velocity $u_{\tau}=\sqrt{\nu \frac{\partial \widetilde{u_{1}}}{\partial y}}$ for which the wall gradient $\left.\frac{\partial \widetilde{u_{1}}}{\partial y}\right|_{y=0}$ is needed. From the data shown in Figure 5 it is apparent that the value 
at the wall location will not yield any meaningful results when the BDIM is employed, which is a common difficulty for IMBMs. However, the value of $\frac{\partial \widetilde{u_{1}}}{\partial y}$ at the fluid boundary $y=\epsilon$ can be used as reasonable approximation for the value at the wall itself due to the law of the wall. Figure 6 compares the streamwise velocity profile scaled in viscous units from simulations obtained with our code and the incompressible reference data provided by Schlatter and Örlü [44]. It can be observed that the body fitted simulation shows slight deviations from the reference in the wake region. This can be attributed to the short domain and inflow length of the current case and the relatively low Reynolds number of $R e_{\theta}=1410$. However, for the purpose of a comparison of the effect of different wall boundary conditions, i.e. bodyfitted and immersed boundary the agreement is reasonable. The comparison of the first order BDIM with the data from the body-fitted case confirms the initial observation of an offset in Figure 5. The effect of this offset leads to considerable deviations from the reference far into the outer region due to the wrong scaling derived from $\frac{\partial \widetilde{u_{1}}}{\partial y}$. However, when the data is scaled with the velocity gradient from the body-fitted simulations the velocity profile matches the reference for $y^{+}>20$ but shows the same offset for $y^{+}<20$. In contrast to that the second order BDIM shows very good agreement throughout the entire profile with some minor overestimates. As for the first order framework the agreement of the case employing the second order correction with the reference is improving further when the profile is scaled by viscous units calculated from the body-fitted simulation.

Figure 7 presents Favre averaged velocity fluctuation profiles scaled in viscous units. The comparison of the body-fitted case and Schlatter's and Örlü's [44] data again shows some minor differences in particular for the streamwise component $\sigma_{11}$. This is due to the reasons noted earlier and for the sake of assessing the BDIM approach the agreement is seen as sufficiently good. The offset in the data obtained with the first order BDIM is prominent in all components and besides the offset all amplitudes are underestimated. The second order framework improves the offset considerably, as already seen for the velocity profile before. The shape of the profile as well as the amplitude is captured well for all components but the streamwise velocity fluctuations $\sigma_{11}$ where the amplitude is overestimated. This overestimation originates from the differences in the exact values of the viscous units used to scale the profiles between the body-fitted and the BDIM cases. As discussed in figure $\left.5 \frac{\partial \widetilde{u_{1}}}{\partial y}\right|_{y=0}$ is approximated with the value at $y=\epsilon$ in 


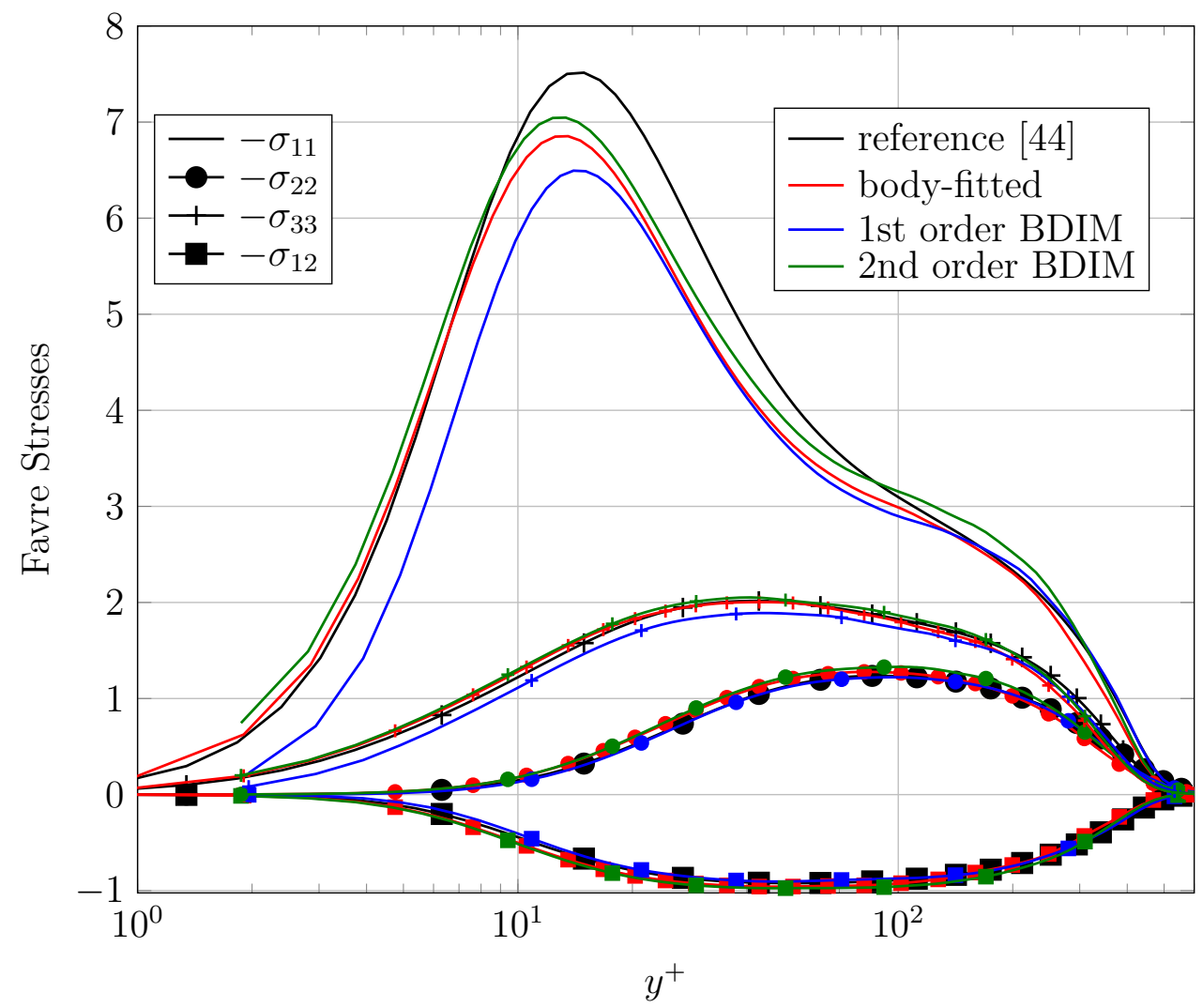

Figure 7: Turbulent boundary layer velocity fluctuations profiles scaled with viscous units comparing the BDIM to the body-fitted boundary conditions and reference data from literature [44]

the cases employing the BDIM. However, when the data obtained from both BDIM simulations is scaled with the same viscous units as the body-fitted case, the deviation in the peak amplitude of the $\sigma_{11}$ component vanishes and overall good agreement is obtained with the reference data.

In the previous paragraphs the surface of the wall was coinciding with the underlying mesh in order to assess the accuracy of the framework when representing a wall adjacent to a turbulent flow. However, the purpose of the BDIM is to represent bodies immersed in fluid flow that do not necessarily coincide with the underlying grid. Therefore two additional simulations have been run where the surface of the plate has an offset in the wall normal 


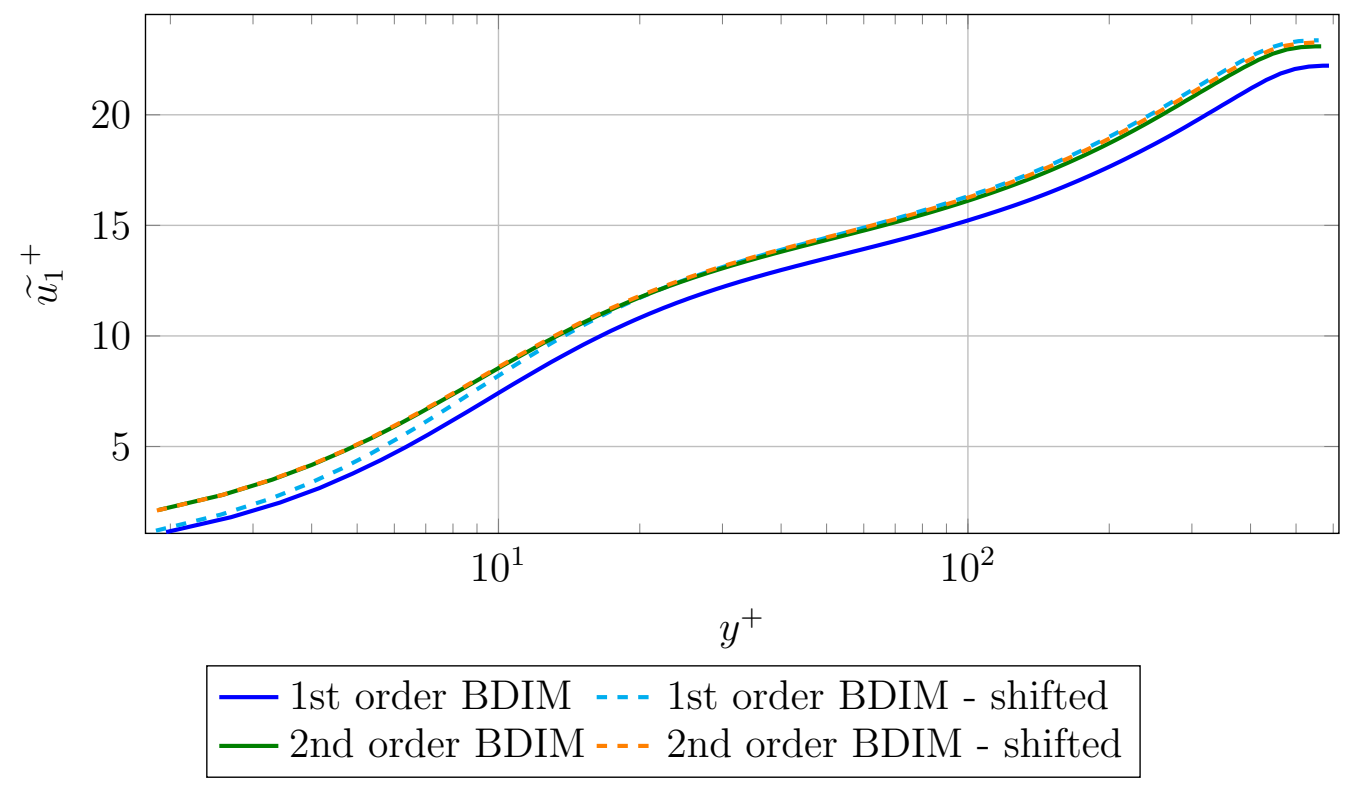

Figure 8: Comparison of the streamwise velocity profile in viscous units of the two BDIM approaches with and without offset in the wall normal direction

direction and is located between the first two grid points of the original grid, i.e. at $y^{+}=0.5$ instead of $y^{+}=0$.

In the following comparison of the cases with and without an offset of the wall location the $y^{+}$coordinates were always corrected accordingly. In figure 8 velocity profiles with and without offset are compared in viscous units. The data from the first order BDIM framework reveals differences between the cases with the two different surface locations from a wall distance of $y^{+} \approx 3$ which are becoming more and more pronounced towards the freestream. In contrast to that, profiles obtained with the second order BDIM with offset are in perfect agreement with data from simulations without offset. That means that the offset increases the uncertainty in the prediction of the velocity gradient at the wall when using the first order BDIM. Indeed the estimated $u_{\tau}$ (calculated from data at $y=\epsilon$ ) changes from $u_{\tau}=0.045$ without offset to $u_{\tau}=0.043$ whereas it does not change in the first two significant digits when employing the second order BDIM with $u_{\tau}=0.43$.

The velocity fluctuations for the same cases are presented in figure 9. As for the mean velocity profile hardly any differences can be observed for the second order BDIM, showing perfect agreement between the cases with and without offset in shape and amplitude of the velocity fluctuations. At the 


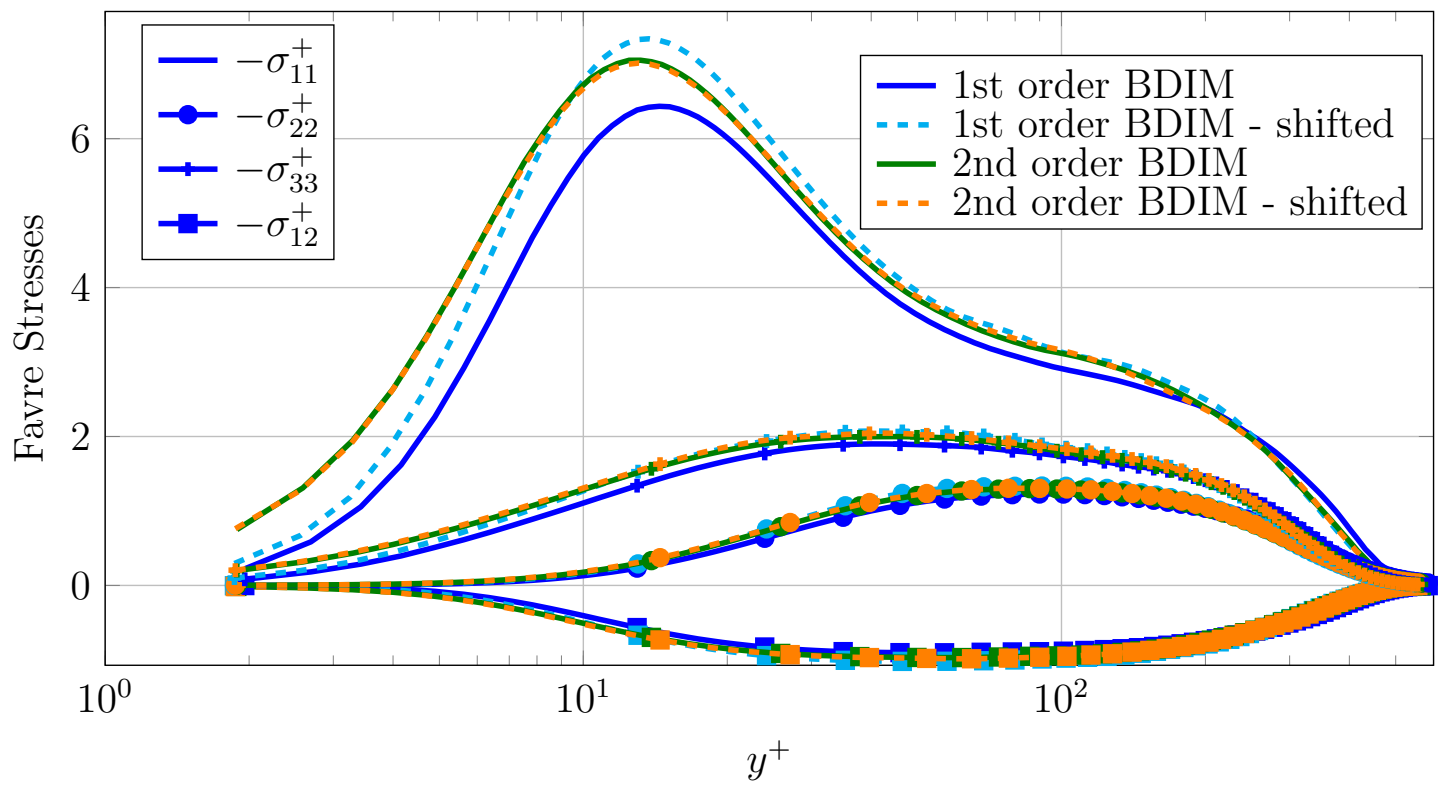

Figure 9: Favre averaged velocity fluctuation profiles in viscous units comparing results with and with out offset in wall normal direction when using the BDIM.

same time significant differences in the streamwise Reynolds stress component $\sigma_{11}^{+}$can be observed.

Overall the BDIM is capable to model the exact surface location very accurately as shown with the mean and fluctuations of the velocity profiles. When the first order framework is employed the velocity gradient at the wall is predicted less accurately and the streamwise velocity fluctuations are overestimated. In contrast to that the results from the second order framework are independent of the exact surface location. Most likely the difference between first and second order can be attributed to the fact that the first order framework hardly uses the full extent of the smoothing region as shown in figure 5 .

Thus it can be concluded that the BDIM approach can be used to accurately represent a wall when wall bounded turbulence is considered. It could be shown that the second order correction improved the results considerably. This can be attributed to the much better representation of the discontinuity present in the velocity gradient at the wall. In addition to that the exact 
location of the plate surface can be represented very accurately leading to a "sharp like" modelling of the surface.

\subsection{Oblique wave growth in a supersonic boundary layer}

When flow induced noise through fluid solid interaction is considered, a very accurate representation of the near-wall flow is crucial. This is attributed to the fact that fluctuations in the boundary layer are the "input" of the noise scattering mechanism. The growth of viscous instabilities, also denoted oblique waves, in a supersonic flat plate boundary layer at $M=3$ is considered as a rigorous test case for the representation of the near-wall physics. This validation problem assesses the correct modelling of the thermodynamic fluctuations close to the wall in particular. In high-fidelity direct noise computations this is an important aspect since the noise is calculated directly from the compressible Navier-Stokes equations.

Oblique waves are three dimensional instabilities that can lead to breakdown into turbulence in supersonic boundary layers. They are considered particularly relevant in low disturbance environments since they need very low disturbance thresholds to be initiated. The oblique breakdown features a linear, an early and a late non-linear regime [45]. For the validation of the BDIM the focus is on simulating the linear regime.

\subsubsection{Numerical Setup}

The flow under consideration is a supersonic flat plate boundary layer at $M=3.0$ and $R e=1,578,102$. In order to trigger the instabilities the flow was disturbed close to the wall by adding a forcing term to the right hand side of the wall normal momentum component in the second spanwise Fourier mode. The amplitude of the volume force was $A=1.0 \times 10^{-3}$ and the frequency was $f=12.558$. The forcing was spread over a circular area in the $x-y$-plane, thus mimicking the effect of a vibrating ribbon. At the inflow boundary the integral formulation of the characteristic boundary condition [29] was used to prescribe a Blasius boundary layer profile with a Reynolds number, based on displacement thickness, of $R e_{\delta^{*}}=273$. At the freestream and outflow boundaries non-reflective characteristic boundary conditions were applied.

For the body-fitted baseline reference case a rectangular Cartesian grid with $1.593 \times 10^{-3}<x<0.456$ and $0.0<y<4.702 \times 10^{-2}$ was employed. In the streamwise direction the 281 grid points were distributed equidistantly 


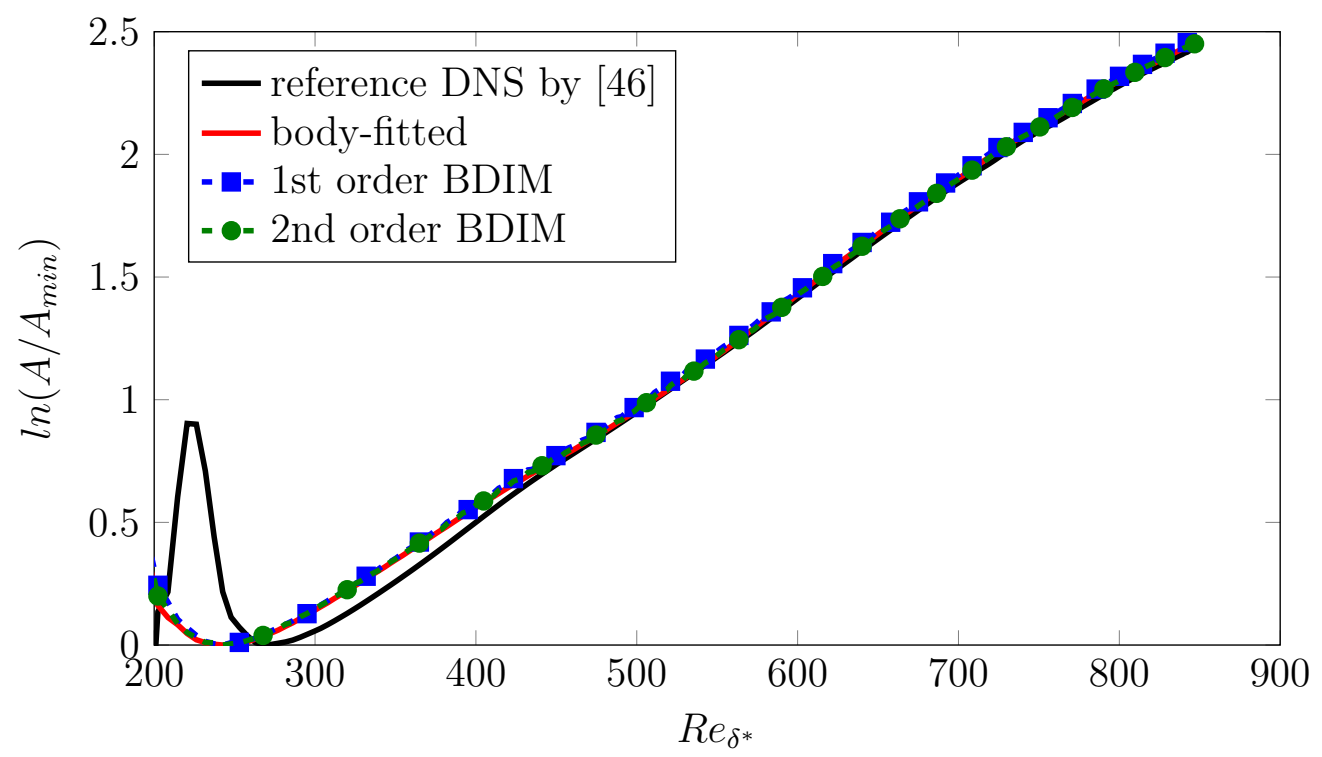

Figure 10: Amplitude of streamwise velocity disturbance as a function of the streamwise position comparing data from [46] with simulations using the BDIM and body-fitted boundary conditions.

with $\Delta x=1.623 \times 10^{-3}$ over the whole domain. In the wall normal direction the grid spacing stretched from $\Delta y=3.510 \times 10^{-5}$ at the wall to $\Delta y=1.644 \times 10^{-3}$ at the freestream boundary. The spanwise direction was discretized with a spectral method using two Fourier modes for a spanwise extent of $\Delta z=0.030$.

In the validation case where the BDIM represents the wall boundary condition, 20 grid points were mirrored in the wall normal direction. The void below the original position of the wall was initialized with zero velocity and uniform density and temperature. The smoothing region half-width $\epsilon$ in units of wall-normal grid spacing in the simulations was $\epsilon=2.0 \Delta y$.

\subsubsection{Results - Accuracy}

After the initial transient instantaneous snapshots of the flow field were gathered for two forcing cycles. From the temporal Fourier transform of the streamwise velocity component the maximum amplitude across the boundary layer was determined as a function of $x$ and then normalized by the lowest amplitude of all streamwise positions during post-processing. Figure $10 \mathrm{com}-$ pares the results obtained with the BDIM to represent the wall with data 


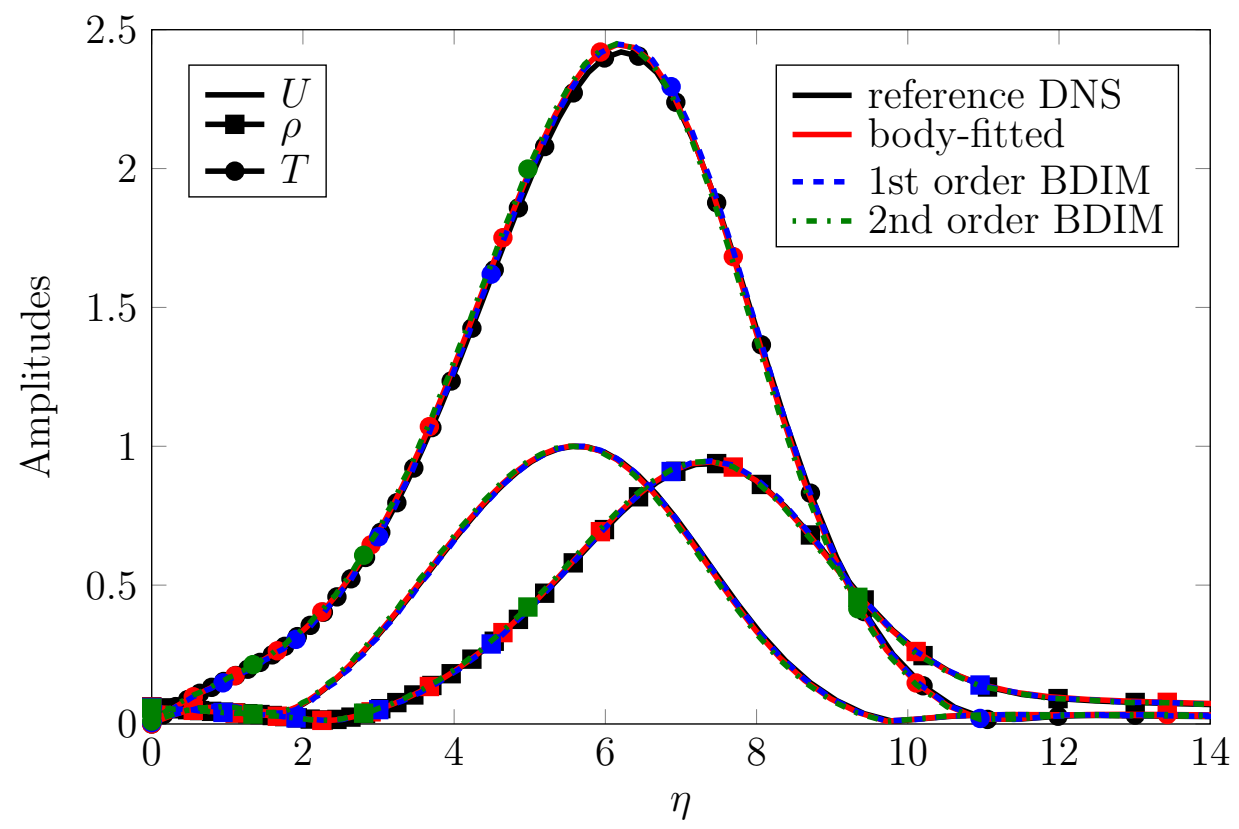

Figure 11: Amplitude distribution of perturbations of streamwise velocity $U$, density $\rho$ and temperature $T$ in the wall-normal direction at $R e_{\delta^{*}}=700$ from the simulations using the BDIM in comparison to simulations with body-fitted boundary conditions and to the results from Husmeier et al. [46]. The wall normal distance $y$ is represented with the similarity variable $\eta=y \sqrt{U /(\nu x)}$, where $U$ is the streamwise velocity component, $\nu$ the viscosity and $x$ the streamwise position. All amplitudes are normalized by the maximum of the velocity amplitude.

using body-fitted boundary conditions with the same code and a reference DNS [46]. It can be noted that there are slight differences between the reference [46] and the body-fitted case in the region of the onset of the instability growth. These can be explained by the fact that the forcing was introduced differently in both cases. Overall it can be appreciated that both the first and second order BDIM reproduce the growth rates very accurately. The averaged deviation in the velocity disturbance amplitude from the body-fitted case for $R e_{\delta^{*}}>300$ is $1.43 \%$ for the first order and $0.48 \%$ for the second order BDIM.

In addition to the instability growth rate, the fluctuation amplitudes of the streamwise velocity $u$, the density $\rho$ and the temperature $T$ are evaluated in figure 11 as a function of the normalized wall distance at $R e_{\delta^{*}}=700$. The amplitudes are normalized by the maximum velocity amplitude. The velocity 


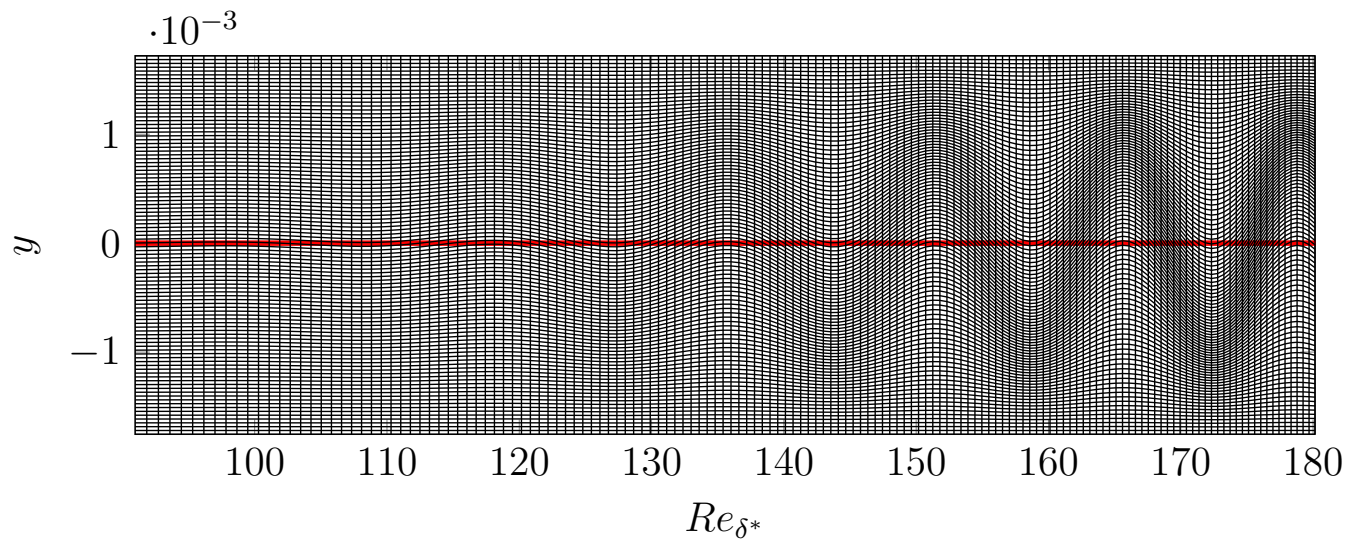

Figure 12: Skewed grid for the oblique wave test case used to validate the method for grids that are not aligned with the surface. Note, the figure only shows part of the streamwise extent of the domain.

amplitude profile proves that the very good agreement between the BDIM and the references is not limited to the maximum amplitude of the velocity fluctuations as presented before. It also matches the shape of the profile along the wall normal direction perfectly. Furthermore the fluctuations of the thermodynamic quantities density $\rho$ and temperature $T$ are computed very accurately. In addition, the normalization that was chosen shows that density fluctuations are almost as high as the velocity fluctuations and the temperature fluctuations are a factor of roughly 2.4 higher that the velocity fluctuations, thus underlining the importance of a good representation of the near wall thermodynamic quantities. Computing these quantities accurately even for low energies is crucial for direct noise computations.

The results presented in the previous paragraphs were obtained using a grid that was aligned with the body surface and the boundary was either represented by body-fitted boundary conditions or the BDIM. To evaluate the accuracy of the BDIM when the boundary is not aligned with the grid lines a new grid, as presented in figure 13, was generated. It was obtained by shifting grid points up to $12 \Delta y_{\text {wall }}$ in the wall normal direction and making this shift sinusoidally dependent on the streamwise location. Towards the inflow and the forcing location the maximum shift was ramped down to zero over 100 grid points. 


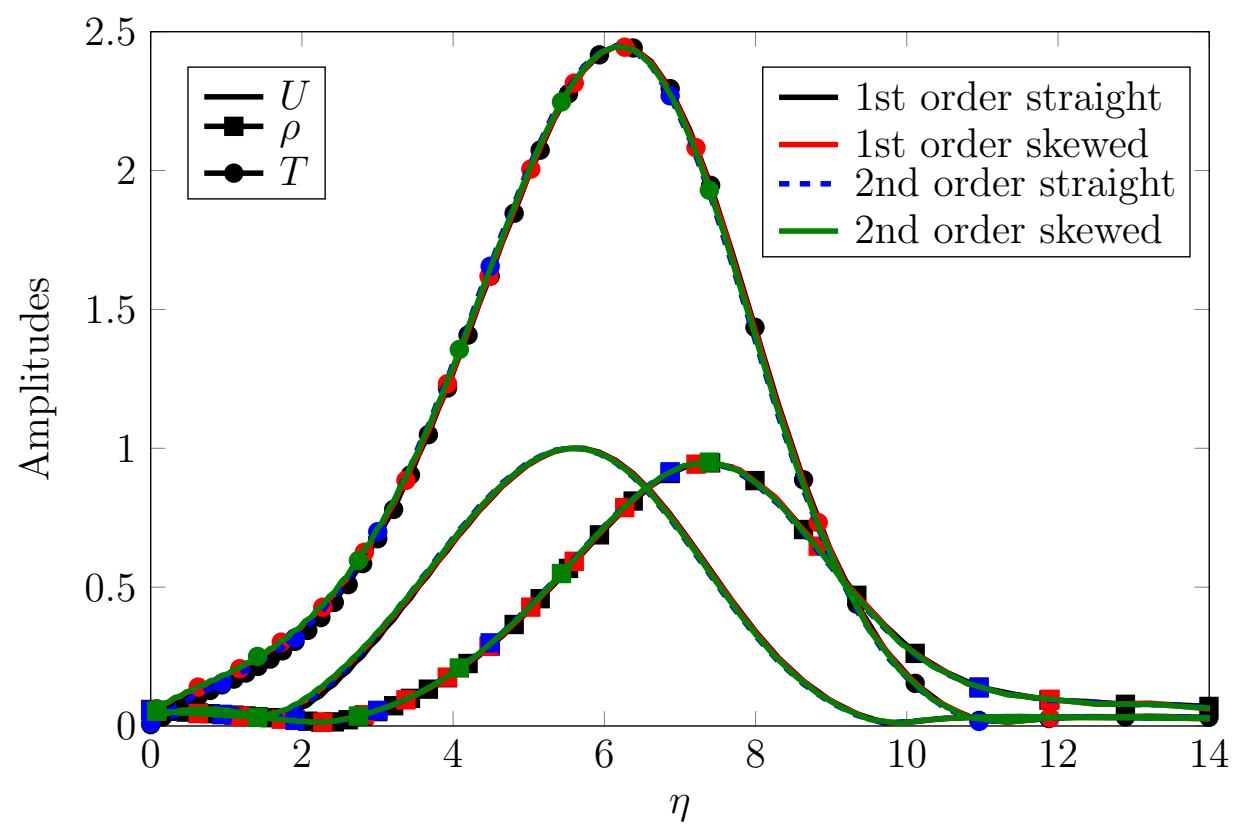

Figure 13: Amplitude distribution of perturbations at $R e_{\delta^{*}}=700$ from the simulations using the BDIM with straight and skewed grids. The wall normal distance $y$ is represented with the similarity variable $\eta=y \sqrt{U /(\nu x)}$, where $U$ is the streamwise velocity component, $\nu$ the viscosity and $x$ the streamwise position. All amplitudes are normalized by the maximum of the velocity amplitude.

Figure 13 compares the thermodynamic fluctuations in the boundary layer between the straight and the skewed grid using the BDIM to represent the wall. The fluctuations of both, the first and the second order BDIM, cases show excellent agreement between straight and skewed grid. When the data is compared quantitatively the largest difference that can be found is in the maximum amplitude of the density fluctuations $\rho$. When using the first order BDIM it is $0.51 \%$ and with the second order BDIM it is $0.43 \%$. For the growth rates, which are not replotted here for conciseness, the difference is slightly higher with $1.75 \%$ for the first and $1.40 \%$ for the second order BDIM.

Overall the results show that the BDIM is capable of accurately representing highly sensitive instability growth adjacent to a wall in a three dimensional compressible flow independent of the underlying grid. 


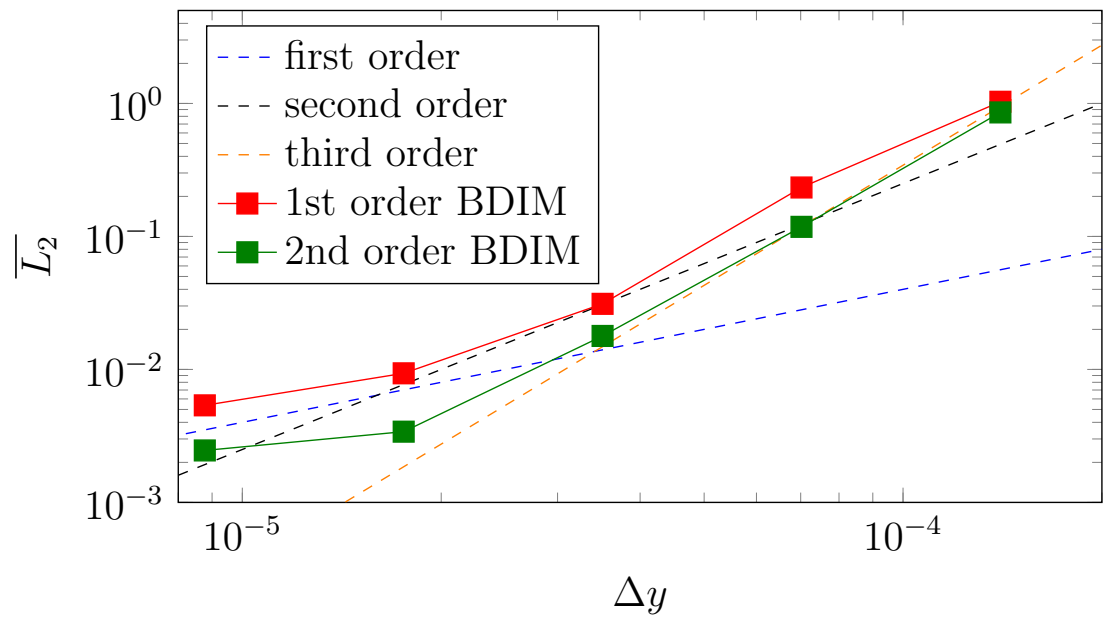

Figure 14: $L_{2}$ convergence of the instability growth rates comparing the first and second order BDIM. A first, second and third order slope are plotted as reference.

\subsubsection{Results - Convergence}

A study with 4 additional resolution levels all together spanning a factor of 16 change in resolution was carried out in order to establish convergence of the instability growth rate for this supersonic case. The results are compared employing the $L_{2}$ norm of the disturbance amplitude in the streamwise extent $300<R e_{\delta^{*}}<800$ relative to the body-fitted reference in Figure 14. The first order BDIM yields a convergence rate that varies between an order of 1.5 and 3 and tails off at the highest resolution. The second order BDIM shows a constant convergence rate that is estimated to be $2.5-3$. It also shows the tail-off at the highest resolution. Overall the error level is lower than in the first order case. From visual inspection of the instantaneous flow field the tail-off is most likely due to a shock downstream of the inflow.

\section{Validation for aeroacoustic problems}

A great advantage of compressible direct numerical simulations is that the generation of flow noise can be investigated without any further modelling as the relevant physical mechanisms are incorporated in the governing equations, i.e. the hydrodynamic and acoustic field are solved simultaneously. The use of the BDIM in such simulations enables the consideration of noise generation by flow interaction with moving objects or bodies with complex 


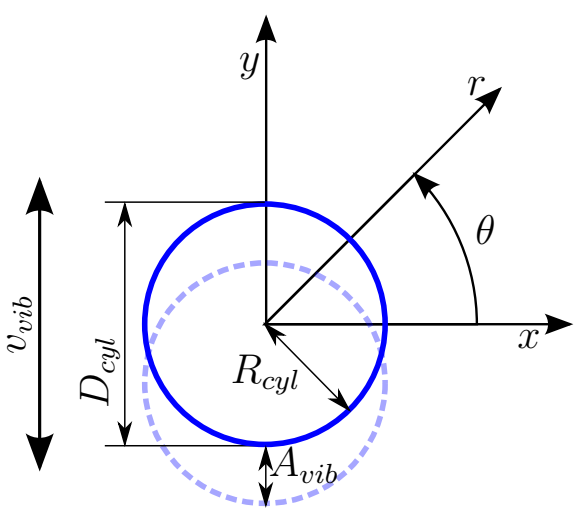

(a) Sketch of the oscillating cylinder.

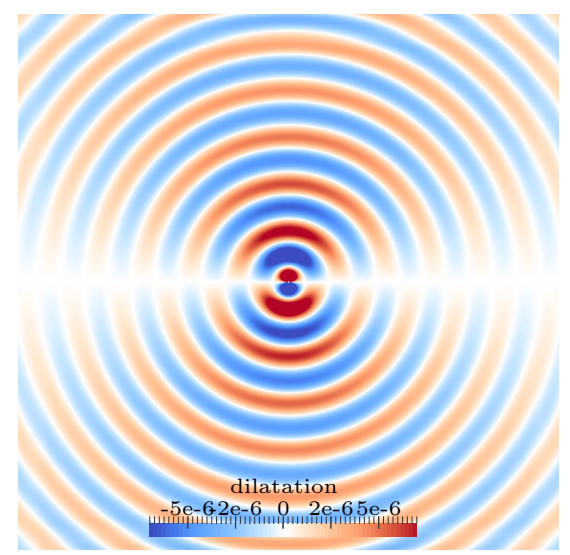

(b) Instantaneous dilatation contours visualizing the acoustic field radiated from the vibrating cylinder.

Figure 15: Introduction of the nomenclature for the physical problem and instantaneous visualization of the acoustic field.

shapes. The following section validates the BDIM for the use in aeroacoustic simulations and considers the noise radiation from a moving body in a medium at rest and trailing-edge noise from an airfoil.

\subsection{Noise radiation from transversely oscillating cylinder}

This section considers the noise radiation from a transversely oscillating cylinder. The cylinder is assumed to have an infinite extension in the spanwise direction and can therefore be simplified to a two dimensional problem. The focus of this case is purely on the noise radiation from a moving body and therefore the surrounding medium of the cylinder is assumed to be at rest.

\subsubsection{Osciallting cylinder - Numerical setup}

The cylinder's diameter $D_{c y l}$ was set to unity and the vibration amplitude $A_{v i b}$ was $5 \%$ of the diameter. The vibration frequency was $f_{v i b}=0.03$ which results in an amplitude for the vibration velocity of $\left|v_{v i b}\right| \approx 9.42 \times 10^{-3}$. With a Mach number of $M=1$ these parameters result in an acoustic wave length of $\lambda_{a}=M / f_{v i b}=33.3 D_{c y l}$. The nomenclature and definition of the parameters is also visualized in figure $15 \mathrm{a}$. The cylinder was discretized with 160 
equispaced grid points per diameter and surrounded by another $1.25 d_{c y l}$ of equispaced grid with the same resolution in each direction. For the transition between the near and the far-field the grid was stretched over 200 grid points to a resolution of 20 grid points per acoustic wave length. The far field of the domain with that resolution covers $6 \lambda_{a}$ approximately. Since the oscillating cylinder is the only noise source and its strength is rather low even minor reflections from the domain boundaries can contaminate the acoustic field. Therefore the outgoing acoustic waves were damped with strong stretching towards the boundaries over an additional 40 grid points in conjunction with the non-reflective boundary conditions.

\subsubsection{Osciallting cylinder - results}

Figure 16 shows the distribution of pressure and skin friction in two different phases during the oscillation cycle. In phase one (figures 16a and 16c) the cylinder is moving with the maximum velocity and passes the origin. The angles $\theta=0^{\circ}$ and $\theta=180^{\circ}$ are on the sides of the cylinder tangential to the direction of motion. The angle of $\theta=90^{\circ}$ is aligned with the direction of motion and the location of the front stagnation point. On the opposite side at $\theta=270^{\circ}$ is the rear stagnation point. It can be appreciated that the pressure distribution presented in figure 16a features two positive peaks at the stagnation points with a higher amplitude at the front stagnation point at an angle of $\theta=90^{\circ}$. At the sides of the cylinder the pressure is reduced as the relative velocity to the fluid is highest here. Overall the first and second order BDIM show good qualitative and quantitative agreement. However, the first order BDIM induces non-physical oscillations at the side of the cylinder. These are eliminated by the use of the derivative correction, i.e. the second order framework. With the distribution of the skin friction coefficient in figure 16c both stagnation points mentioned earlier can be confirmed with the zero-crossing at $\theta=90^{\circ}$ and $\theta=270^{\circ}$. At the sides of the cylinder the amplitude of the skin friction is highest as the relative velocity to the fluid is highest at these locations. Both BDIM frameworks show a very smooth distribution and can hardly be distinguished.

In phase two (figure $16 \mathrm{~b}$ and 16d) the cylinder is at the location with the maximum displacement from the original position and the velocity is zero before the direction of motion is reversed. The pressure distribution presented in figure $16 \mathrm{~b}$ is symmetric with ambient pressure at the sides of the cylinder, i.e. at $0^{\circ}, 180^{\circ}$, and features symmetric peaks on the sides that 


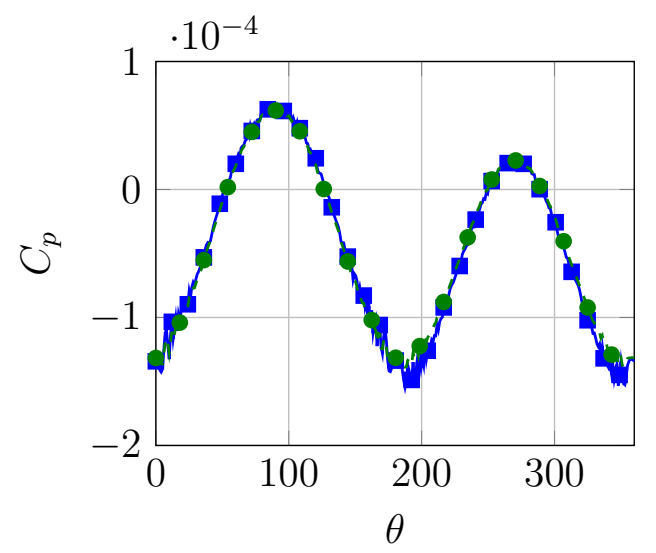

(a) pressure coefficient distribution phase one

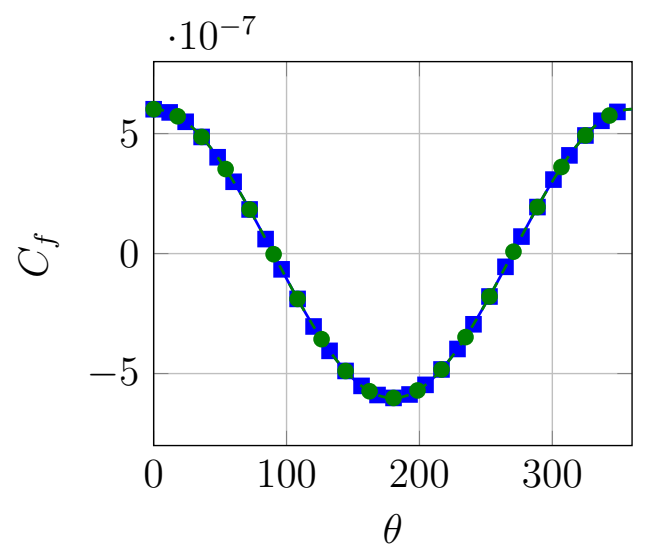

(c) skin friction coefficient distribution phase one

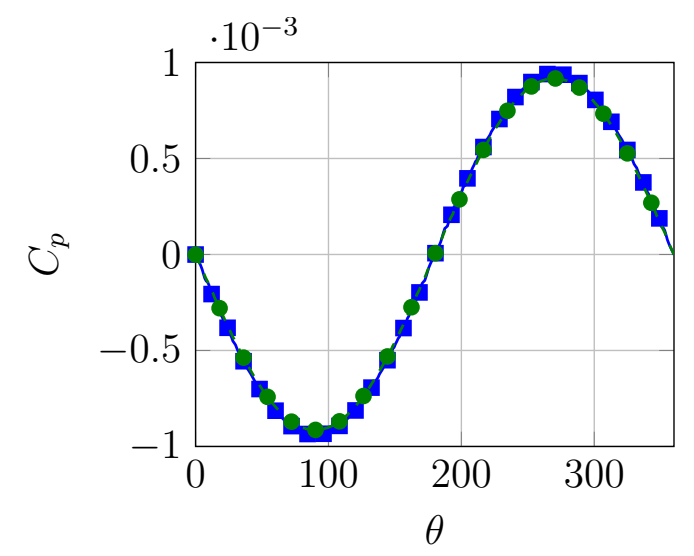

(b) pressure coefficient distribution phase two

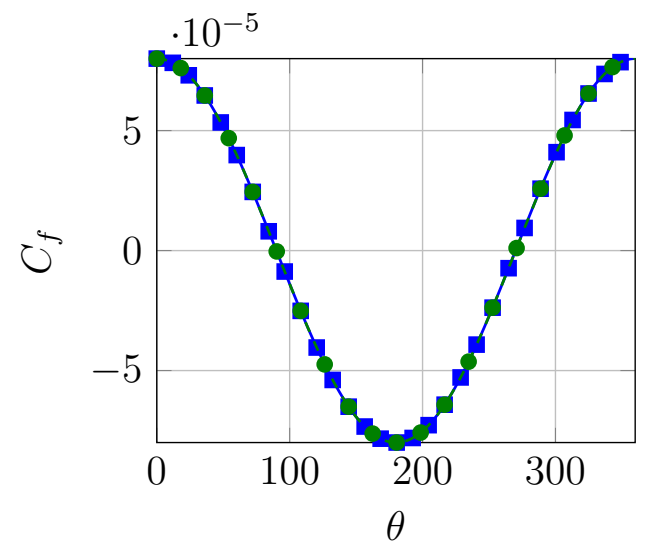

(d) skin friction coefficient distribution phase one

$\rightarrow-1$ st order BDIM - -2 nd order BDIM

Figure 16: Pressure and skin friction coefficient distribution around cylinder during oscillation. In phase one the cylinder is moving with the maximum velocity and phase two is when the velocity is zero and the direction of motion is reversed.

are aligned with the direction of motion, i.e. $90^{\circ}$ and $270^{\circ}$. In the direction the cylinder was moving in the previous phase, i.e. $90^{\circ}$, the pressure is lowered compared to the ambient pressure and the opposite is true for the rear stagnation point of the previous phase, i.e. $270^{\circ}$. Both amplitudes are approximately an order of magnitude higher than in phase one with a very 
smooth distribution. This can be explained with the motion induced flow field that leads to a comparably high relative velocity between cylinder and fluid, despite the fact that the cylinder is not moving in this phase. The two peak amplitudes are slightly higher in the first order framework. In the skin friction distribution presented in figure $16 \mathrm{~d}$ it is found that the higher relative velocity leads to a skin friction that is two orders of magnitude higher than in previous phase.

The analytical solution for the pressure fluctuations $p_{r m s}$ of this radiation problem is

$$
p_{r m s}=\frac{1}{\sqrt{2}}\left|-j \rho_{0} v_{v i b} \cos (\theta) \frac{H_{1}^{(2)}\left(k_{a} r\right)}{M H_{1}^{(2)^{\prime}}\left(k_{a} R_{c y l}\right)} e^{j 2 \pi f_{v i b} t}\right|,
$$

where $H_{1}^{(2)}$ is a Hankel function of the second kind and first order and $H_{1}^{(2)^{\prime}}$ its derivative with the wave number $k_{a}=1 / \lambda_{a}$ as part of its argument. Furthermore $\rho_{0}$ is the mean density of the medium at rest [47]. The near field approximation can be expressed by

$$
p_{r m s}=\frac{1}{\sqrt{2}}\left|-j 2 \pi f_{v i b} \rho_{0} v_{v i b} R_{c y l}^{2} M^{2} \frac{1}{r}\right| \quad,
$$

and the far field approximation is

$$
p_{r m s}=\frac{1}{\sqrt{2}}\left|2 \pi^{2} f_{v i b}^{1.5} \rho_{0} R_{c y l}^{2} v_{v i b} M^{1.5} \frac{1}{\sqrt{r}}\right| .
$$

Figure 15b presents the dilatation contours at an arbitrary instance of time from the simulation with the first order BDIM. The dilatation contours show the expected dipole like radiation pattern with no signs of any spurious oscillations from either the domain boundaries or the BDIM. This is confirmed by the averaged directivity of the radiated sound pressure levels (SPL) in Figure 17a. The data is extracted from the acoustic far-field at a radial distance of $r=2 \lambda_{a}$. It can be appreciated that the shape of the directivity pattern simulated by the BDIM is in excellent agreement with the analytical reference. The amplitudes of the two simulations employing the BDIM agree reasonably well with the reference but overestimate the noise 


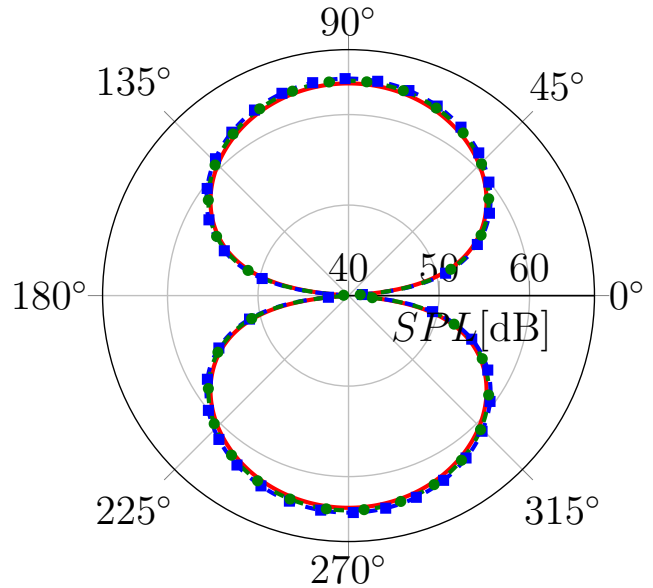

(a) Directivity of noise radiated from the cylinder at a radial distance of $r=2 \lambda_{a}$ or $r=132 D_{c y l}$. For the legend refer to the neighboring figure.

Figure 17: Quantitative validation comparing the data from the simulation to the analytical reference.

level by $0.53 \mathrm{~dB}$ for first order and $0.29 \mathrm{~dB}$ for the second order BDIM at this resolution level of $\Delta x=\Delta y=D_{c y l} / 160$.

Figure 17b compares the SPL as a function of the radial distance of the two simulations with the analytical solution. The data was extracted along the radial direction aligned with the cylinder's motion. The findings from the SPL directivity are confirmed in that the simulations employing the BDIM slightly overestimate the SPL by a constant value. It is apparent that the second Order BDIM agrees better with the reference than the first order approach. Table 2 compares the averaged deviation from the analytical reference along the main radiation direction for the first and second order BDIM for different resolution levels. The deviation from the analytical solution is a factor of 1.39 to 1.92 higher when the first order BDIM is employed compared to the second order. Even for the highest resolution the deviation from the analytical solution is not vanishing for either case. It is argued that the simulation converges to a different solution as is also suggested by the constant offset found in the previous discussion. The most likely reason for that is the assumption of an inviscid fluid in the derivation of the analytical 


\begin{tabular}{cccc}
\hline \hline$N_{p t s} / D_{c y l}$ & $A_{v i b} / \Delta x$ & 1st Ord BDIM $\Delta S P L[\mathrm{~dB}]$ & 2nd Ord BDIM $\Delta S P L[\mathrm{~dB}]$ \\
\hline 20 & 1 & 2.91 & 1.64 \\
40 & 2 & 1.64 & 0.88 \\
80 & 4 & 0.92 & 0.48 \\
160 & 8 & 0.53 & 0.29 \\
320 & 16 & 0.34 & 0.21 \\
640 & 32 & 0.25 & 0.18 \\
& & & \\
\hline \hline
\end{tabular}

Table 2: Summary of deviation from the analytical reference for different resolution levels using the first and second order BDIM .

solution. However, the simulations do have a finite viscosity. An additional test simulation showed that when the viscosity is increased by two orders or magnitude the deviation between the analytical solution and the simulated result decreased to $\triangle S P L=0.47 \mathrm{~dB}$ on the $N_{p t s} / D_{c y l}=80$ grid using the second order framework. When viscosity was reduced by two orders of magnitude this deviation grew to $\triangle S P L=1.72 \mathrm{~dB}$ on the same grid and with the same method. The action of viscosity adds an additional component of fluid acceleration tangential to the translation direction.

The formal order of convergence of the data presented in table 2 is visualized in figure 18. The data is referenced against the case with the highest resolution. It is obvious that the first and second order BDIM converge with the same rate of approximately two for the cases with higher resolution. The major difference between the two is the level of the deviation, as discussed before. The fact that both cases yield the same rate of convergence is not surprising. As shown with the results from the turbulent boundary layer in Figure 5, the main effect of the second order correction is the improved representation of the wall velocity gradient in the wall bounded shear flow. In the current case considering an oscillating cylinder in a medium at rest the shear is very small and hence the contribution of the derivative correction term of equation 7 is small.

In summary the overall agreement with the analytical reference is excellent considering the very low energies that are involved in this acoustic test case. This validation case demonstrates that the BDIM is an appropriate 


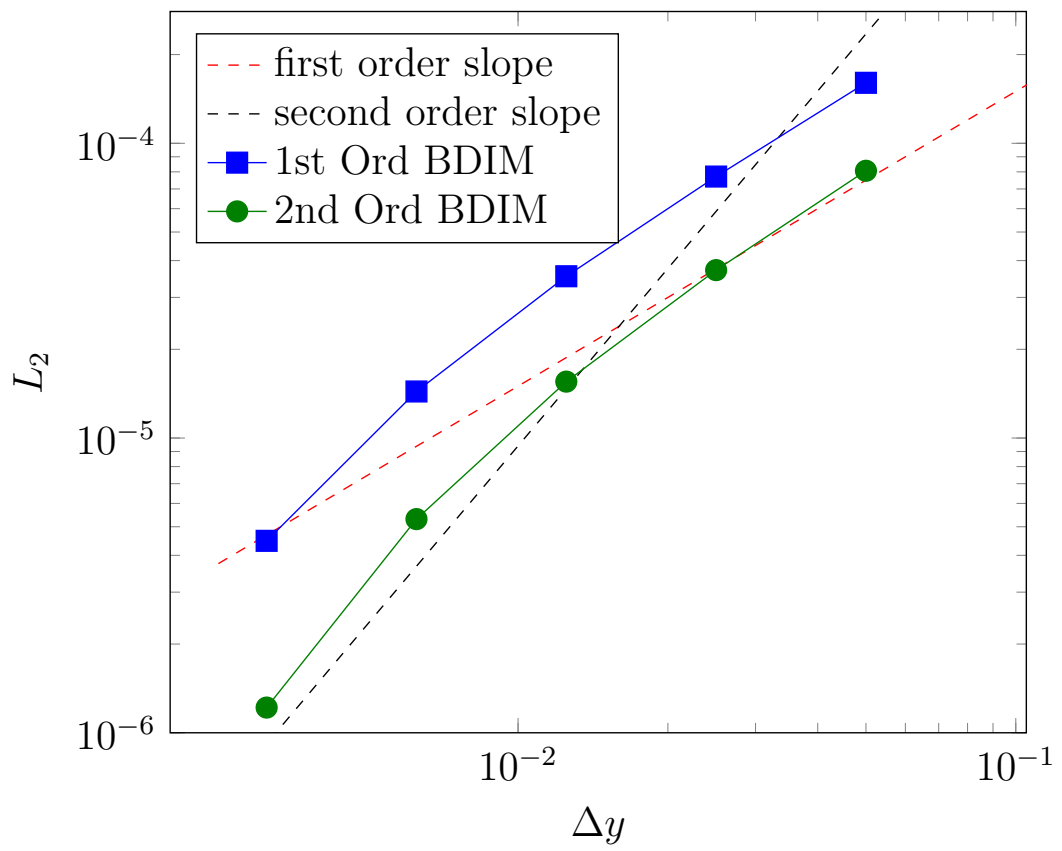

Figure 18: Convergence of the pressure fluctuations relative to the analytical reference over the lowest grid spacing $\Delta y$ for the first and the second order BDIM.

method to perform high-fidelity simulations featuring noise radiation from moving bodies.

\subsection{Noise radiation from an airfoil with a trailing-edge extension}

We next consider trailing-edge noise from a trailing-edge extension of an airfoil at angle of attack. In this validation case the aeroacoustic noise generation is caused by the interaction of a stationary body with the unsteady flow around it. The noise radiation from the trailing-edge will be the strongest source of noise in this case as the interaction of pressure fluctuations in the flow with the rigid trailing-edge increases the efficiency of the radiation process.

\subsubsection{Airfoil trailing-edge noise - Numerical setup}

The flow parameters of this case are the chord based Reynolds number $R e_{c}=5 \times 10^{4}$, Mach number $M=0.4$ and angle of attack $A o A=5^{\circ}$. At these flow conditions the flow separates on the suction side and forms a laminar separation bubble as can be seen in the spanwise vorticity contours 


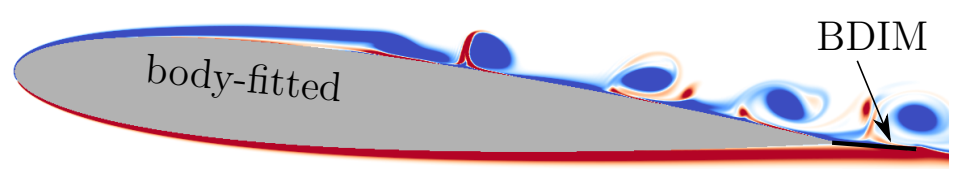

(a) Snapshot of the spanwise vorticity field indicating where the BDIM is employed and the separation on the suction side.

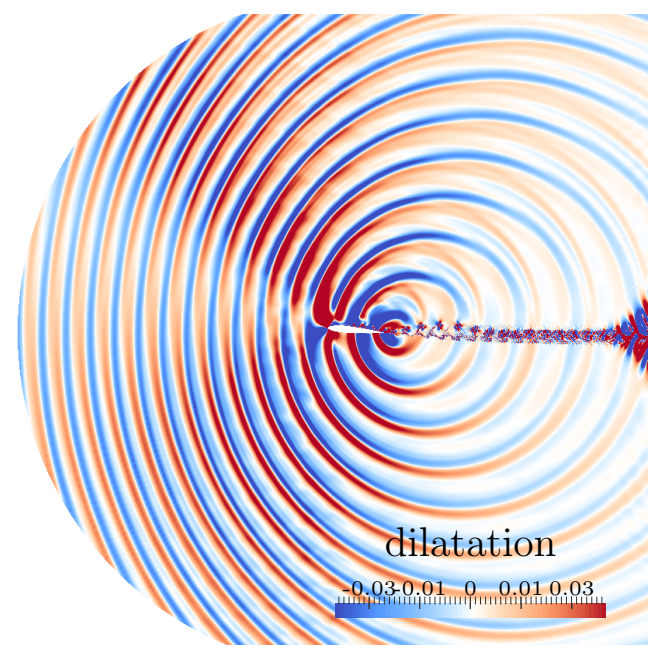

(b) Dilatation contours of a simulation where the 1st order BDIM is employed to represent the the airfoil's extension. The spread of the wake close to the outflow origniates from the action of the zonal boundary condition.

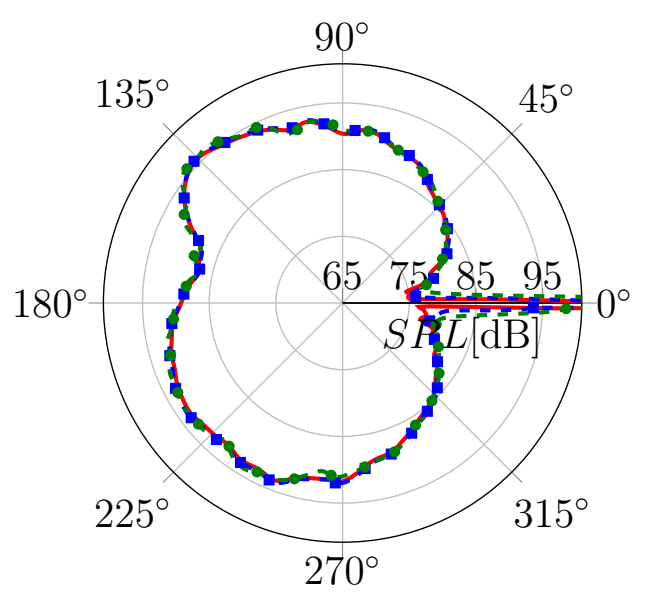

(c) Noise levels at a radial distance of $r=4$ chord lengths comparing simulation results with body-fitted boundary conditions $(-)$ and the first (---) and second (---) order BDIM.

Figure 19: Body-fitted airfoil simulation where the extension is represented by the BDIM.

presented in figure 19a. The computational grid was derived from the grid G3 in Jones et al. [48]. In order to optimize the resolution close to the airfoil surface and reduce numerical cost the airfoil body is represented by a body conforming grid and body-fitted boundary conditions. As indicated in figure 19a the trailing-edge extension was represented by the BDIM. This hybrid approach reduces the computational cost significantly but leaves the flexibility to change the shape of the extension or make it an actuated moving control surface. 


\subsubsection{Airfoil trailing-edge noise - Results}

The acoustic field of this case is visualized with contours of dilatation in figure 19b. It can be seen that the noise originates from the trailing-edge of the extension and travels predominantly in the upstream direction. The acoustic field is very clean and shows no signs of spurious oscillations from the domain boundaries or the BDIM.

Figure 19c compares the noise directivity when the flat plate extension is represented by either body-fitted boundary conditions and by the BDIM. This plot shows excellent agreement between the two cases employing the BDIM and the body-fitted boundary conditions for the noise level as well as for the directivity shape. This demonstrates that the first and second order BDIM are able to represent the acoustic scattering of pressure disturbances from a trailing-edge accurately.

\section{Conclusion}

The compressible extension of the Boundary Data Immersion Method originally developed for incompressible flows [18] has been introduced. The compressible formulation uses the BDIM meta equation to map the velocity and temperature fields between a solid body and a fluid subdomain. For certain applications such as the simulation of bluff bodies the proposed mapping of the continuity equation as an interface condition between fluid and solid can enhance robustness of the simulation.

A thorough validation of the novel compressible BDIM framework showed that the second order extension increases accuracy of the simulations compared to the first order approach. In particular the discontinuity of the velocity gradient at the wall is modelled much more accurately. This is shown for the very challenging case of a fully turbulent boundary layer where the discontinuity of the velocity gradient is relatively high and a broad range of scales in time and space is present in the flow.

The case of a transversely oscillating cylinder in a medium at rest showed that the BDIM is able to predict the noise radiation from a moving body with high accuracy. Furthermore flow induced noise generated by the interaction of a solid body with unsteady fluid flow can be modelled accurately with the BDIM. To the authors' knowledge this is the first virtual boundary method that has been validated for direct noise simulations considering moving bodies. 
There is no penalty in allowable time step compared to body-fitted simulations when using the BDIM. Also the grid resolution does not need to be higher than in an equivalent body-fitted simulation to accurately reproduce the compressible flow physics given that the grid is aligned with the immersed surface. In all cases considered the second order correction reduced the solution error and improved the convergence rate. Hence the BDIM for compressible flows offers a computationally efficient yet accurate approach to represent stationary or moving bodies in high-fidelity numerical simulations with application to aeroacoustics. The method has already been employed successfully for fluid-structure interaction problems [49]. We also envision it to be appropriate for aero-vibro-acoustic studies and other applications that require accurate representation of arbitrary moving boundaries.

\section{Acknowledgement}

The first author acknowledges the faculty for Engineering and the Environment of the University of Southampton for funding his PhD. Furthermore this work used the ARCHER UK National Supercomputing Service

(http://www.archer.ac.uk) for which computational time was provided through the UK turbulence consortium, EPSRC grant number EP/L000261/1 and is gratefully acknowledged.

\section{AppendixA. Weighting functions at the intersection of two planes}

In Section 2, kernel moments are analytically evaluated in order to accurately immerse the boundary data from the solid mechanical system onto the fluid mechanical equations of motion. In the appendix, the case of a geometry with sharp corners (which requires special care) is locally treated as the intersection of two component planar geometries.

First we use Eq 6a to determine the moment at point $\vec{x}$ of each of the component planes $a$ and $b$, and call them $\mu_{0, a}, \mu_{0, b}$. We name these planes such that $\mu_{0, a} \leq \mu_{0, b}$, ie $\vec{x}$ is further into the fluid relative to $a$. In the case that $x$ is not near the intersection, the moment of the shape $c$ defined as the intersection of $a$ and $b$ is simply the moment of $a$,

$$
\mu_{0, c}=\mu_{0, a}
$$

However, when $\vec{x}$ is in the smoothing region of both $a$ and $b$, such that $0<\mu_{0, a} \leq \mu_{0, b}<1$, then both planes contribute to the compound moment. 
An approximate value for the compound moment in this case is

$$
\mu_{0, c}=\mu_{0, a} \mu_{0, b}^{\log _{2} \frac{\pi}{\theta}}
$$

subject to the bound

$$
\mu_{0, c} \geq \max \left(0, \mu_{0, a}+\mu_{0, b}-1\right)
$$

We see that the angle $\theta$ between $a$ and $b$ determines how heavily $\mu_{0, b}$ influences the compound moment. For $\theta=\pi$, the compound shape is flat and there is no change to Eq A.1. For $\theta<\pi$, the shape is a sharp corner, and the moment decreases sharply as you approach the intersection of the two planes. The bound ensures that the compound moment is still accurate for very small angles when the kernel regions of the two planes do not overlap.

Once this moment is established, it implicitly defines the effective distance $d_{c}$ and normal $\hat{n}_{c}$ of the compound shape. If $\mu_{0, c}$ is given by Eq A.1 then $d_{c}=d_{a}$ and $\hat{n}_{c}=\hat{n}_{a}$. Otherwise, the distance $d_{c}$ is found by inverting Eq 6a. Note that the bounds on $\mu_{0, c}$ ensure we only need the first line of Eq 6a. Let us call the inverse of the Eq $6 \mathrm{a} h$, ie

$$
d(\vec{x})=h\left(\mu_{0}(\vec{x})\right)
$$

Then $d_{c}=h\left(\mu_{0, c}\right)$ is used in Eq $6 \mathrm{~b}$ to find the effective first moment $\mu_{1, c}$ without issue.

The normal $\hat{n}$ is defined as

$$
\hat{n}(\vec{x})=\vec{\nabla} d(\vec{x})=h^{\prime}\left(\mu_{0}\right) \vec{\nabla} \mu_{0}(\vec{x})
$$

where

$$
h^{\prime}=\frac{\partial h}{\partial \mu_{0}}
$$

and we note that $h^{\prime}$ only scales the magnitude not the direction of the normal. First, let's assume that the lower bound Eq A.3 is active, then the normal is proportional to

$$
\hat{n}_{c} \propto \vec{\nabla} \mu_{0, a}+\vec{\nabla} \mu_{0, b}=\frac{\hat{n}_{a}}{h^{\prime}\left(\mu_{0, a}\right)}+\frac{\hat{n}_{b}}{h^{\prime}\left(\mu_{0, b}\right)}
$$

where Eq A.4 is used to substitute the normal for each component. We see 
the effective normal is a weighted sum of the component normals.

Next, using Eq A.2, applying the chain rule, and substituting the normals for each component as before gives

$$
\hat{n}_{c} \propto \frac{1}{\mu_{0, a}} \frac{\hat{n}_{a}}{h^{\prime}\left(\mu_{0, a}\right)}+\frac{\log _{2}\left(\frac{\pi}{\theta}\right)}{\mu_{0, b}} \frac{\hat{n}_{b}}{h^{\prime}\left(\mu_{0, b}\right)}
$$

where again we see that the angle $\theta$ determines the relative influence of plane $b$.

\section{References}

[1] M. de Tullio, P. De Palma, G. Iaccarino, G. Pascazio, M. Napolitano, An immersed boundary method for compressible flows using local grid refinement, Journal of Computational Physics 225 (2007) 2098-2117.

[2] R. Mittal, G. Iaccarino, Immersed boundary methods, Annual Review of Fluid Mechanics 37 (2005) 239-261.

[3] A. Chaudhuri, A. Hadjadj, A. Chinnayya, On the use of immersed boundary methods for shock/obstacle interactions, Journal of Computational Physics 230 (2011) 1731-1748.

[4] P. Tran, F. Plourde, Computing compressible internal flows by means of an Immersed Boundary Method, Computers \& Fluids 97 (2014) 21-30.

[5] D. Casalino, P. di Francescantonio, Y. Druon, GFD Predictions of Fan Noise Propagation, in: 10th AIAA/CEAS Aeroacoustics Conference, AIAA Paper 2004-2989, 2004.

[6] R. Arina, An Immersed Boundary Method for Aeroacoustics Problems, in: 14th AIAA/CEAS Aeroacoustics Conference, AIAA Paper 20083003, 2008.

[7] M. Cand, A. Sayma, M. Imregun, 10th AIAA/CEAS Aeroacoustics Conference, in: 10th AIAA/CEAS Aeroacoustics Conference, AIAA Paper 2004-2816, 2004.

[8] M. Liu, K. Wu, Aerodynamic noise propagation simulation using immersed boundary method and finite volume optimized prefactored compact scheme, Journal of Thermal Science 17 (4) (2008) 361-367. 
[9] J. H. Seo, R. Mittal, A High-Order Immersed Boundary Method for Acoustic Wave Scattering and Low-Mach Number Flow-Induced Sound in Complex Geometries., Journal of Computational Physics 230 (4) (2011) 1000-1019.

[10] F. Margnat, Hybrid prediction of the aerodynamic noise radiated by a rectangular cylinder at incidence, Computers \& Fluids 109 (2015) 13-26.

[11] D. Goldstein, G. Handler, L. Sirovich, Modeling a no-slip flow boundary with an external force field, Journal of Computational Physics 105 (1993) 354-366.

[12] P. De Palma, M. de Tullio, G. Pascazio, M. Napolitano, An immersedboundary method for compressible viscous flows, Computers \& Fluids 35 (2006) 693-702.

[13] R. Ghias, R. Mittal, H. Dong, A sharp interface immersed boundary method for compressible viscous flows, Journal of Computational Physics 225 (2007) 528-553.

[14] Q. Liu, O. Vasilyev, A Brinkman penalization method for compressible flows in complex geometries, Journal of Computational Physics 227 (2007) 946-966.

[15] R. Sandberg, L. Jones, Direct numerical simulations of low Reynolds number flow over airfoils with trailing-edge serrations, Journal of Sound and Vibration 330 (2011) 3818-3831.

[16] O. Hu, N. Zhao, J. Liu, A Ghost Cell Method for Turbulent Compressible Viscous Flows on Adaptive Cartesian Grids, Procedia Engineering 67 (2013) 241-249.

[17] G. Weymouth, D. Yue, Boundary data immersion method for Cartesiangrid simulations of fluid-body interaction problems, Journal of Computational Physics 230 (2011) 6233-6247.

[18] A. Maertens, G. Weymouth, Accurate Cartesian-grid simulations of near-body flows at intermediate Reynolds numbers, Computer Methods in Applied Mechanics and Engineering 283 (2015) 106-129. 
[19] J. Yang, E. Balaras, An embedded-boundary formulation for large-eddy simulation of turbulent flows interacting with moving boundaries, Journal of Computational Physics 215 (2006) 12-40.

[20] R. Mittal, H. Dong, M. Bozkurttas, F. Najjar, A. Vargas, A. von Loebbecke, A versatile sharp interface immersed boundary method for incompressible flows with complex boundaries, Journal of Computational Physics 227 (2008) 4825-4852.

[21] J. Seo, R. Mittal, A Sharp-Interface Immersed Boundary Method with Improved Mass Conservation and Reduced Spurious Pressure Oscillations, Journal of Computational Physics 230 (2011) 7347-7363.

[22] H. Luo, H. Dai, P. Ferreira de Sousa, B. Yin, On the numerical oscillation of the direct-forcing immersed-boundary method for moving boundaries, Computers \& Fluids 56 (2012) 61-76.

[23] C. Liu, C. Hu, An efficient immersed boundary treatment for complex moving object, Journal of Computational Physics 274 (2014) 654-680.

[24] W. Breugem, A second-order accurate immersed boundary method for fully resolved simulations of particle-laden flows, Journal of Computational Physics 231 (2012) 4469-4498.

[25] M. H. Carpenter, J. Norstrom, D. Gottlieb, A stable and conservative interface treatment of arbitrary spatial accuracy, Journal of Computational Physics 148 (1999) 341-365.

[26] C. Kennedy, M. Carpenter, R. Lewis, Low-storage, explicit RungeKutta schemes for the compressible NavierStokes equations, Applied Numerical Mathematics 35 (2000) 177-219.

[27] C. Kennedy, A. Gruber, Reduced aliasing formulations of the convective terms within the Navier-Stokes equations for a compressible fluid, Journal of Computational Physics 227 (2008) 1676-1700.

[28] C. Bogey, N. de Cacqueray, C. Bailly, A shock-capturing methodology based on adaptative spatial filtering for high-order non-linear computations, Journal of Computational Physics 228 (2009) 1447-1465. 
[29] L. Jones, Numerical studies of the flow around an airfoil at low Reynolds number, Ph.D. thesis, School of Engineering Sciences University of Southampton (2008).

[30] R. Sandberg, N. Sandham, Nonreflecting zonal characteristic boundary condition for direct numerical simulation of aerodynamic sound, AIAA Journal 44 (2) (2006) 402-405.

[31] J. Park, K. Kwon, H. Choi, Numerical solutions of flow past a circular cylinder at Reynolds numbers up to 160, KSME International Journal 12 (6) (1998) 1200-1205.

[32] R. Henderson, Details of the drag curve near the onset of vortex shedding, Physics of Fluids 7 (1995) 2102-2104.

[33] H. Zhang, U. Fey, B. Noack, M. Konig, H. Eckelmann, On the transition of the cylinder wake, Physics of Fluids 7 (4) (1995) 779.

[34] J.-I. Choi, R. C. Oberoi, J. R. Edwards, J. A. Rosati, An immersed boundary method for complex incompressible flows, Journal of Computational Physics 224 (2007) 757-784.

[35] P. Chiu, R. Lin, T. Sheu, A differentially interpolated direct forcing immersed boundary method for predicting incompressible NavierStokes equations in time-varying complex geometries, Journal of Computational Physics 229 (2010) 4476-4500.

[36] D. Russell, Z. Wang, A Cartesian grid method for modeling multiple moving objects in 2D incompressible viscous flow, Journal of Computational Physics 191 (2003) 177-205.

[37] C. Norberg, An experimental investigation of the flow around a circular cylinder: influence of aspect ratio, Journal of Fluid Mechanics 258 (1994) $287-316$.

[38] D. J. Tritton, Experiments on the flow past a circular cylinder at low Reynolds numbers, Journal of Fluid Mechanics 6 (1959) 547-567.

[39] M. Pourquie, Accuracy close to the wall of immersed boundary methods, in: 4th European Conference of the International Federation for Medical and Biological Engineering, 2009, pp. 1939-1942. 
[40] E. Saiki, S. Biringen, Numerical simulation of a cylinder in uniform flow: application of a virtual boundary method, Journal of Computational Physics 123 (1996) 450-465.

[41] M. Klein, A. Sadiki, J. Janicka, A digital filter based generation of inflow data for spatially developing direct numerical or large eddy simulations, Journal of Computational Physics 186 (2003) 652-665.

[42] E. Touber, N. Sandham, Large-eddy simulation of low-frequency unsteadiness in a turbulent shock-induced separation bubble, Theoretical and Computational Fluid Dynamics 23 (2009) 79-107.

[43] P. Schlatter, R. Örlü, Assessment of direct numerical simulation data of turbulent boundary layers, Journal of Fluid Mechanics 659 (2010) $116-126$.

[44] P. Schlatter, R. Örlü, Turbulent boundary layers at moderate Reynolds numbers: inflow length and tripping effects, Journal of Fluid Mechanics 710 (2012) 5-34.

[45] C. Mayer, D. von Terzi, H. Fasel, Direct numerical simulation of complete transition to turbulence via oblique breakdown at Mach 3, Journal of Fluid Mechanics 674 (2011) 5-42.

[46] F. Husmeier, C. Mayer, H. Fasel, Investigation of Transition of Supersonic Boundary Layers at Mach 3 Using DNS, in: 43th AIAA Aerospace Sciences Meeting and Exhibit, 2005.

[47] D. Blackstock, Fundamentals of Physical Acoustics, John Wiley \& Sons, 2000.

[48] L. Jones, R. Sandberg, N. Sandham, Direct numerical simulations of forced and unforced separation bubbles on an airfoil at incidence, Journal of Fluid Mechanics 602 (2008) 175-207.

[49] S. Serrano Galliano, R. Sandberg, Effect of the leading and trailing edge geometry on the fluid-structural coupling of membrane aerofoils, in: 2016 AIAA SciTech, AIAA Paper 2016-0853, 2016. 